\title{
3-INTERVAL IRREDUCIBLE PARTIALLY ORDERED SETS
}

\author{
STEFAN FELSNER
}

\begin{abstract}
In this paper we discuss the characterization problem for posets of interval dimension at most 2 . That is, we attempt to compile the minimal list of forbidden posets for interval dimension 2. Members of this list are called 3interval irreducible posets. The problem is related to a series of characterization problems which have been solved earlier. These are: The characterization of planar lattices, due to Kelly and Rival [KeRi75], the characterization of posets of dimension at most 2 (3-irreducible posets) which has been obtained independently by Trotter and Moore [TrMo76] and by Kelly [Ke77] and the characterization of bipartite 3-interval irreducible posets due to Trotter [Tr81].

We show that every 3-interval irreducible poset is a reduced partial stack of some bipartite 3-interval irreducible poset. Moreover, we succeed in classifying the 3-interval irreducible partial stacks of most of the bipartite 3-interval irreducible posets. Our arguments depend on a transformation $P \rightarrow B(P)$, such that $\operatorname{Idim} P=\operatorname{dim} B(P)$. This transformation has been introduced in [FHM91].
\end{abstract}

\section{InTRODUCTION AND BASICS}

An extension of a poset $P=\left(X,<_{P}\right)$ is a partial order $Q=\left(X,<_{Q}\right)$ on the same set, that contains all the relations of $P$, i.e., $x<_{P} y$ implies $x<_{Q} y$. A family $\left\{Q_{1}, \ldots, Q_{k}\right\}$ of extensions of $P$ is said to realize $P$ if for all $x, y \in X$ we have $x<_{P} y$ exactly if $x<_{Q_{i}} y$ for all $1 \leq i \leq k$. If we restrict the $Q_{i}$ to belong to a special class of orders and seek for a minimum size realizer we come up with a concept of dimension.

A linear extension of $P=(X,<)$ is an extension $L=\left(X,<_{L}\right)$ of $P$ which is a chain, i.e., $x<_{L} y$ or $y<_{L} x$ for all $x, y \in X$ with $x \neq y$. Dushnik and Miller [DuMi41] defined the (order) dimension of a poset $P$, denoted $\operatorname{dim}(P)$, as the smallest integer $k$ for which there exist $k$ linear extensions realizing $P$.

A partial order $P$ is an interval order if it can be represented by assigning an interval $I_{x}=\left(a_{x}, b_{x}\right)$ on a chain $C$ to each element $x \in P$, such that $x<y$ in $P$ iff $b_{x}<a_{y}$ in $C$. Fishburn [Fi85] characterized interval orders as the posets with no 4 -point

1991 Mathematics Subject Classification. 06A07,05C35.

Key words and phrases. Partially ordered sets, interval orders, dimension.

supported by the DFG under grant FE 340/2-1 
subset forming two disjoint 2 -chains, i.e., no $\mathbf{2 + 2}$. The interval dimension of $P$, denoted $\operatorname{Idim}(P)$, is the smallest $k$ for which there exist $k$ interval extensions of $P$ which realize $P$. Since linear orders are interval orders we obtain the trivial inequality $\operatorname{Idim}(P) \leq \operatorname{dim}(P)$.

If $P^{\prime}=\left(X^{\prime},<\right)$ is a suborder of $P=(X,<)$, then $\operatorname{dim}\left(P^{\prime}\right) \leq \operatorname{dim}(P)$ and $\operatorname{Idim}\left(P^{\prime}\right) \leq$ $\operatorname{Idim}(P)$. Therefore, for every integer $k$ there is a minimal list of posets of dimension (interval dimension) $k+1$, such that every poset which does not contain a subposet from this list has dimension (interval dimension) at most $k$. Members of this list then are called $(k+1)$-irreducible $((k+1)$-interval irreducible $)$ posets. Note that a $(k+1)$ irreducible $((k+1)$-interval irreducible) poset $P$ has $\operatorname{dim}(P)=k+1(\operatorname{Idim}(P)=k+1)$ but the removal of any element lowers its dimension (interval dimension).

The list of 2-irreducible posets only contains the 2 element antichain and the list of 2 -interval irreducible posets only contains the $\mathbf{2}+\mathbf{2}$. The list of 3 -irreducible posets is of a much higher complexity. Counting only one of $P$ and $P^{d}$, the dual of $P$, the list consists of 10 isolated examples together with 7 infinite families (see Figure 1). This list has been obtained independently by Trotter and Moore [TrMo76] and by Kelly [Ke77]. Since this paper depends on the ideas of Kelly's argument we now give a brief (and in some details not even correct) outline of his proof.

Let $\mathbf{L}(P)$ denote the completion by cuts of a poset $P$, i.e., $\mathbf{L}(P)$ is the smallest lattice containing $P$ as a suborder. Kelly's characterization of the 3 -irreducible posets is mainly based on two results:

(1) $\operatorname{dim} P=\operatorname{dim} \mathbf{L}(P)$. (A theorem of Baker).

(2) The complete list $\mathcal{L}$ of lattices of dimension 3 with the property that every proper sublattice is of lower dimension. (Let us remark that a lattice is planar exactly if it contains no sublattice from $\mathcal{L}$ ). The list $\mathcal{L}$ had been obtained by Kelly and Rival [KeRi75].

Suppose that $P$ is a 3 -irreducible poset. It follows that $\operatorname{dim} \mathbf{L}(P) \geq 3$, therefore, $\mathbf{L}(P)$ contains one or more of the lattices in $\mathcal{L}$ as sublattices. The key idea for the proof is to consider the lattices in $\mathcal{L}$ in a specific ordering, Kelly order. At an intermediate step in the argument we assume that $\mathbf{L}(P)$ contains a lattice $Q$ from this list, but does not contain any lattice preceding it in the Kelly order. The details of each individual case center around chosing a copy of a 3-irreducible subposet $R$ of $Q$ and then one by one showing how those points of $R$ which do not belong to $P$ can be replaced by points of $P$.

A poset $P=(X,<)$ is called bipartite if there are two sets $X_{1}, X_{2} \subseteq X$, such that $x<y$ implies $x \in X_{1}$ and $y \in X_{2}$. Trotter found a transformation, which associates with a bipartite poset $P$ a poset $\operatorname{Stack}(P)$, such that, $\operatorname{Idim}(P)=\operatorname{dim} \operatorname{Stack}(P)$. This fact, together with the existing list of 3-irreducible posets lead to the characterization of bipartite 3 -interval irreducible posets in [Tr81]. 
Figure 1. The 3 irreducible orders (part 1)

B

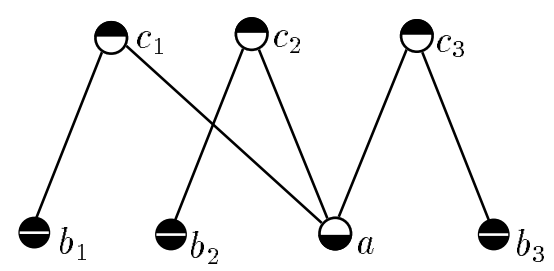

D

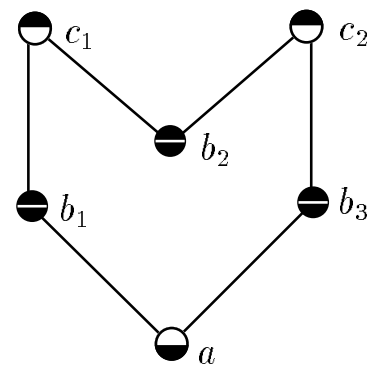

$\mathrm{CX}_{2}$

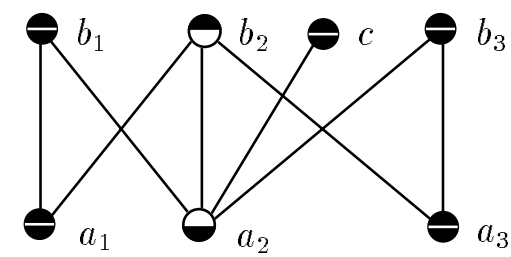

$\mathrm{EX}_{1}$

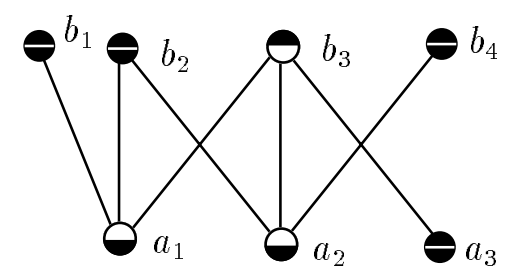

$\mathrm{FX}_{1}$

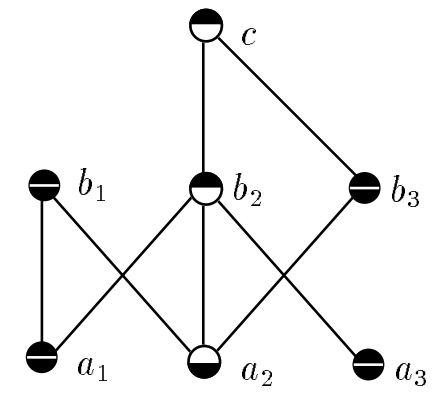

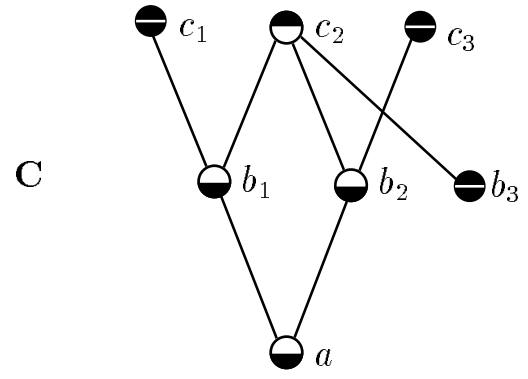
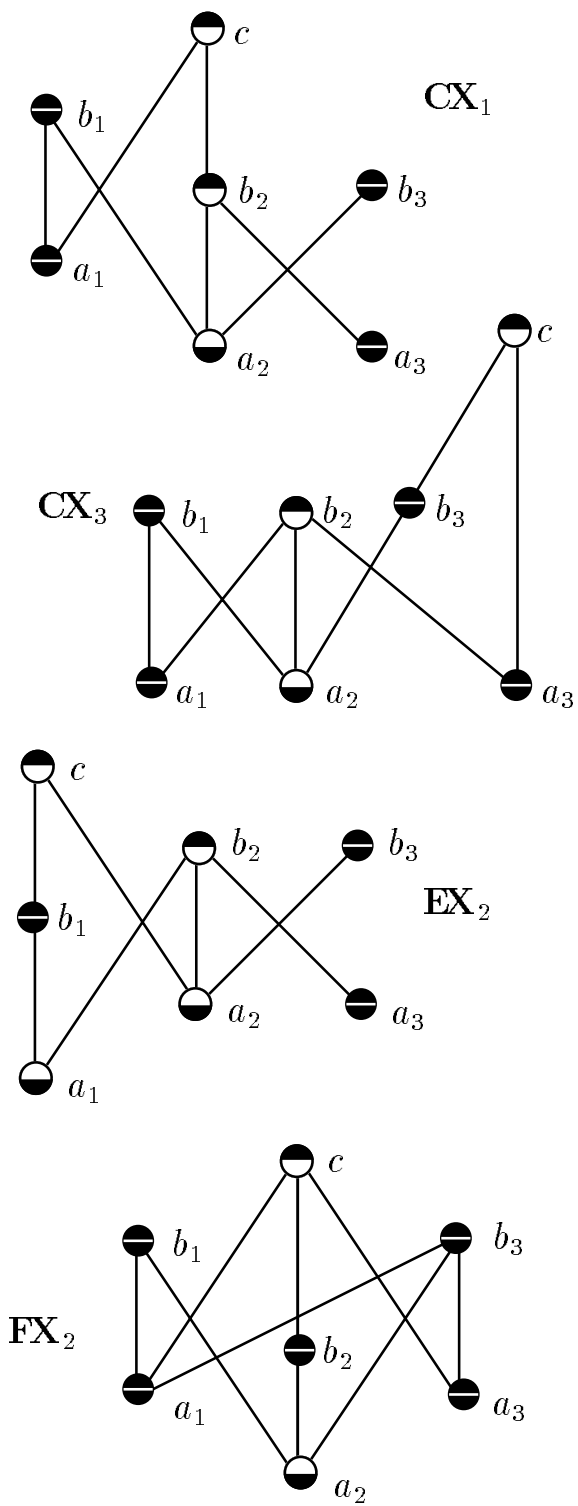

represents $\ell$-labeled elements

- represents elements that are $\ell$ - and $u$-labeled

- represents $u$-labeled elements 
Figure 1. The 3 irreducible orders (part 2)

$\mathbf{A}_{n}$
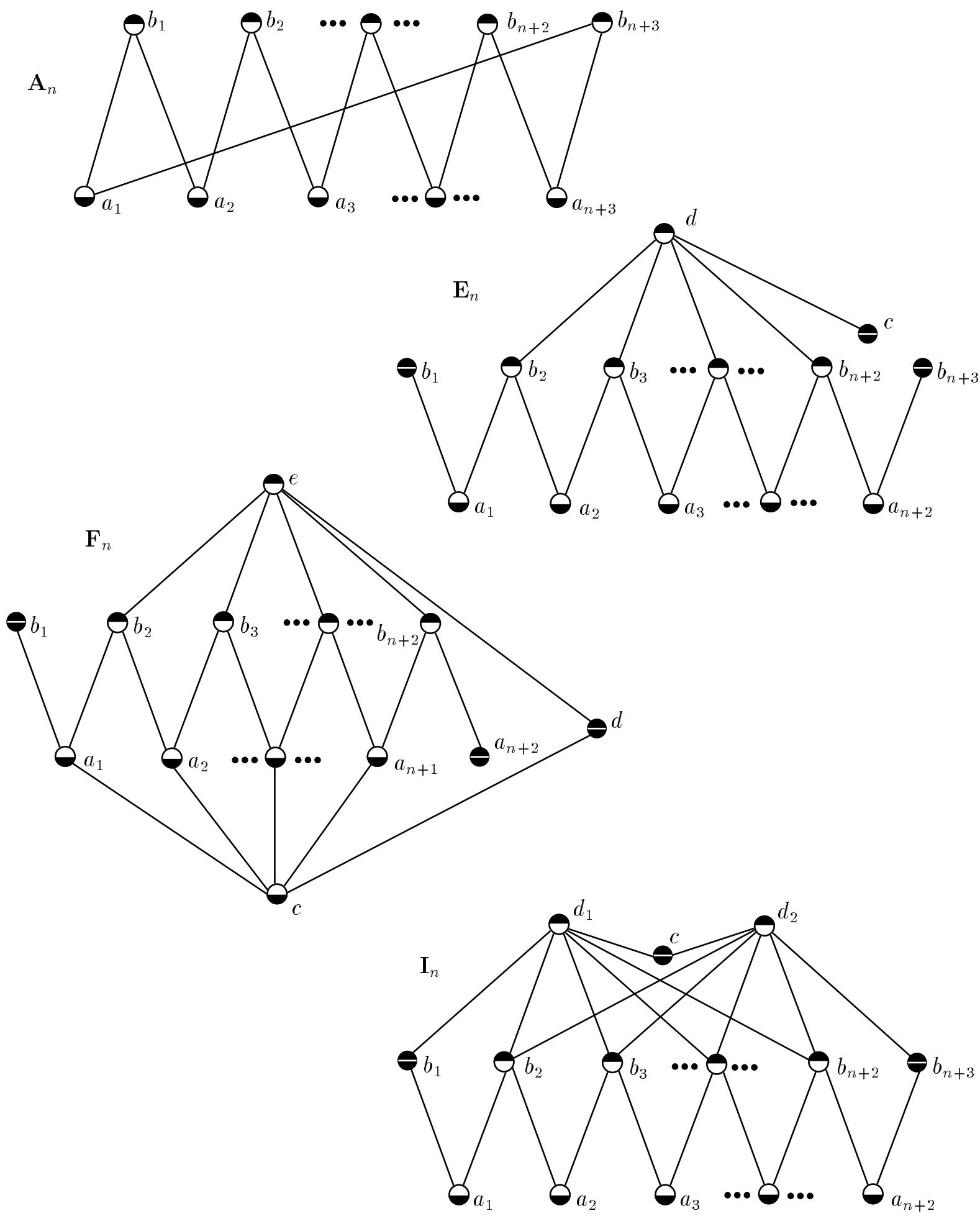
Figure 1. The 3 irreducible orders (part 3 )
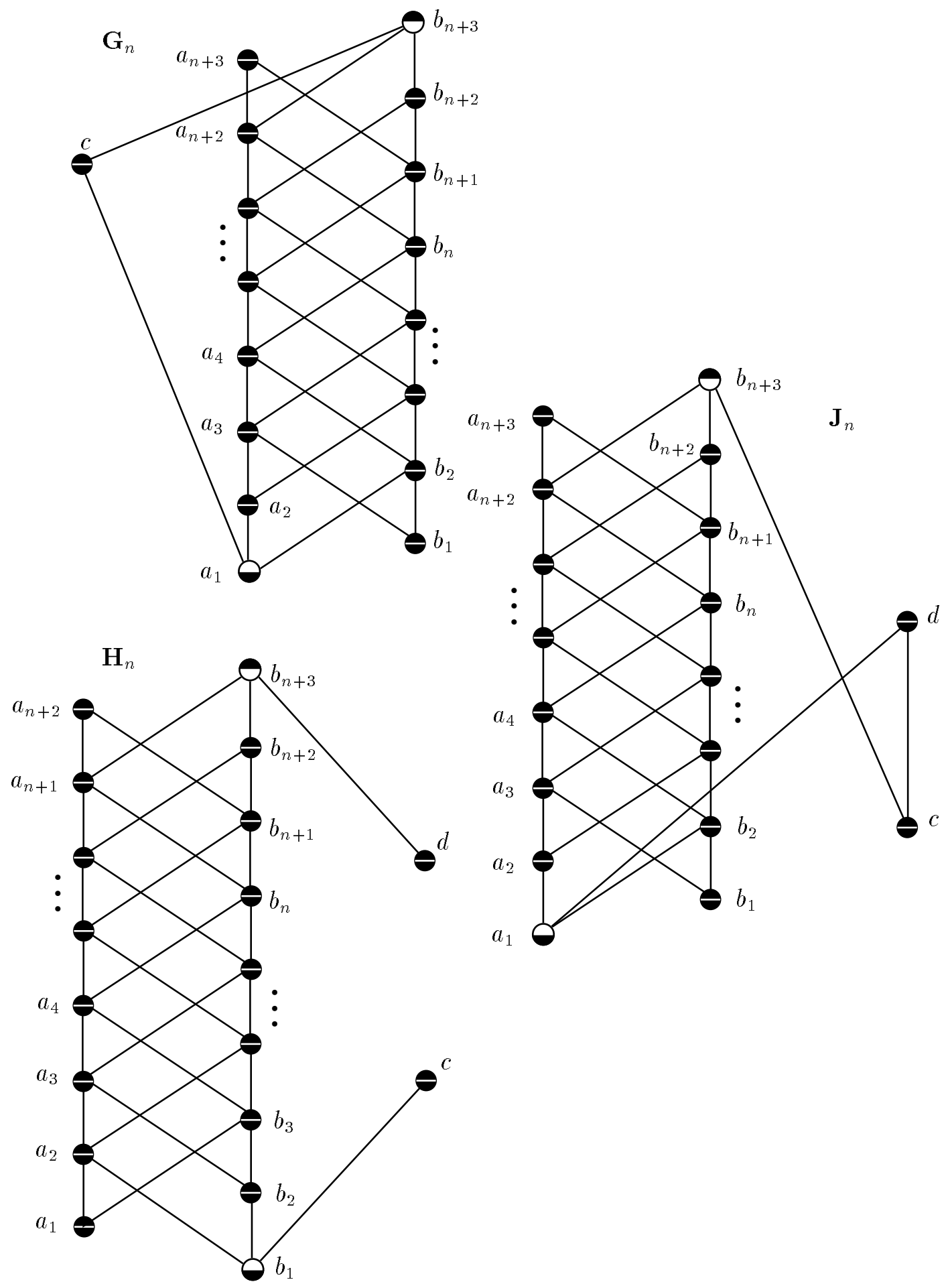
Trotter's proof uses Kelly's approach for the characterization of the 3-irreducible posets as a road map. Since the stack of a bipartite 3 -interval irreducible poset $P$ contains one (or more) of the 3 -irreducible posets we can argue about the implications for the original poset. Again, a specific ordering of the 3 -irreducible posets and corresponding assumptions about the 3 -irreducible posets contained in Stack $(P)$ organize the proof.

In [FHM91] we have introduced a transformation $P \rightarrow B(P)$, such that $\operatorname{Idim} P=$ $\operatorname{dim} B(P)$ for arbitrary posets $P$. In this paper we use this operator $P \rightarrow B(P)$, to approach the characterization of 3 -interval irreducible posets. The argument is again based on a Kelly ordering of the 3 -irreducible posets.

Let $P=(X,<)$ be a poset, for $x \in X$ we denote the predecessor set $\{y \in X: y<x\}$ of $x$ with $\operatorname{Pred}(x)$, the successor set $\{y \in X: y>x\}$ is denote by $\operatorname{Succ}(x)$. The closed predecessor set is $\operatorname{Pred}[x]=\{y \in X: y \leq x\}$, i.e., Pred $[x]=\operatorname{Pred}(x) \cup\{x\}$, similarly $\operatorname{Succ}[x]=\operatorname{Succ}(x) \cup\{x\}$. We now review the definition and some properties of the transformation $P \rightarrow B(P)$.

Definition 1. For each element $x$ of a poset $P=(X,<)$ let

$$
\begin{aligned}
& L(x)=\operatorname{Pred}(x), \\
& U(x)=\bigcap_{z \in \operatorname{Succ}(x)} \operatorname{Pred}(z), \text { if } \operatorname{Succ}(x)=\emptyset \text { then let } U(x)=X .
\end{aligned}
$$

With $P$ we associate a poset $B(P)=(Y,<)$. The elements of $Y$ are the distinct sets occurring as $L(x)$ or $U(x)$ for some $x \in X$. The ordering of $B(P)$ is given by setinclusion.

The next definition is taken from [Mi92].

Definition 2. For posets $P=\left(X,<_{P}\right)$ and $Q=\left(Y,<_{Q}\right)$ we say that $P$ has an interval representation on $Q$ if there are mappings $L: X \rightarrow Y$ and $U: X \rightarrow Y$, such that

(1) $L(x)<_{Q} U(x)$, i.e, $[L(x), U(x)]$ is a nondegenerate interval of $Q$ for each $x \in X$.

(2) $U\left(x_{1}\right) \leq_{Q} L\left(x_{2}\right)$ exactly if $x_{1}<_{P} x_{2}$.

An important fact about interval representations is given with the next lemma.

Lemma 1. If an order $P$ has an interval representation on some poset $Q$, then $\operatorname{Idim} P \leq \operatorname{dim} Q$.

The next theorem collects properties of the transformation $P \rightarrow B(P)$.

Theorem 1. Let $P=(X,<)$ and $B(P)=(Y,<)$.

(1) The mappings $L: X \rightarrow Y$ and $U: X \rightarrow Y$ define an interval representation of $P$ on $B(P)$.

(2) The dimension of $B(P)$ equals the interval dimension of $P$. 
We now come to the stack of a bipartite poset. As mentioned, the stack played a central role in the characterization of bipartite 3 -interval irreducible posets.

Definition 3. Let $P=\left(X,<_{P}\right)$ be a (connected) bipartite poset and let $O$ be an arbitrary linear order on $X$. Let $A$ denote the set of minimal elements and $B$ the set of maximal elements of $P$ (we assume that $a, a_{i} \in A$ and $b, b_{i} \in B$ ). The $\operatorname{Stack}(P)$ is a extension of $P$, the relations of $\operatorname{Stack}(P)$ are given by

$$
\begin{array}{ll}
a<b & \text { if } a<b \text { in } P, \\
b_{1}<b_{2} & \text { if } \operatorname{Pred}_{P}\left(b_{1}\right) \subset \operatorname{Pred}_{P}\left(b_{2}\right), \\
a_{1}<a_{2} & \text { if } \operatorname{Succ}_{P}\left(a_{1}\right) \supset \operatorname{Succ}_{P}\left(a_{2}\right), \\
b<a & \text { if } a \| b \text { and } a^{\prime}<b^{\prime} \text { in } P \text { for all } a^{\prime} \in \operatorname{Pred}_{P}(b) \text { and } b^{\prime} \in \operatorname{Succ}_{P}(a), \\
b_{1}<b_{2} & \text { if } \operatorname{Pred}_{P}\left(b_{1}\right)=\operatorname{Pred}_{P}\left(b_{2}\right) \text { and } b_{1}<_{O} b_{2}, \\
a_{1}<a_{2} & \text { if } \operatorname{Succ}_{P}\left(a_{1}\right)=\operatorname{Succ}_{P}\left(a_{2}\right) \text { and } a_{1}<_{O} a_{2} .
\end{array}
$$

We now show, that for a bipartite order $P$ the posets $B(P)$ and $\operatorname{Stack}(P)$ are intimately related. Let the 0,1-closure of $P$, i.e., the adjoin of a least element 0 and a greatest element 1 , be denoted by $P \rightarrow \widehat{P}$.

Theorem 2. If $P=(X,<)$ is a bipartite order, then $B(P)=\left.\widehat{\operatorname{Stack}(P)}\right|_{\sim}$, here $\sim$ is an equivalence relation on $X$ with the properties:

- each class of $\sim$ is an autonomous subset of $\operatorname{Stack}(P)$,

- Stack $(P)$ induces a chain on each class of $\sim$.

Proof. An element $x \in X$ is mapped to two elements of $B(P)$. Since $P$ is bipartite we either have $L(x)=\emptyset$ or $U(x)=X$. In the first case let $M(x)=U(x)$, in the second case let $M(x)=L(x)$. Also, let $O$ be the linear order used in the construction of Stack $(P)$. Define a poset $Q$ on $X$ by

(1) $x<_{Q} y$ if $M(x) \subset M(y)$,

(2) if $M(x)=M(y)$ then $x<_{Q} y$ if either $x \in \operatorname{Min}(P)$ and $y \in \operatorname{Max}(P)$ or $x$ precedes $y$ in $O$.

Note that $B(P)$ is the 0,1 -closure of the poset $(\{M(x) \mid x \in X\}, \subset)$. Therefore $B(P)=\widehat{\left.Q\right|_{\sim}}$ when $x \sim y$ iff $M(x)=M(y)$.

We claim that $Q=\operatorname{Stack}(P)$. The proof is an easy case analysis using the definitions of $L(x), U(x)$ and the definition of the Stack. Let $A=\operatorname{Min}(P)$ and $B=\operatorname{Max}(P)$.

$a<b$ in $P$ : In $\operatorname{Stack}(P)$ we have $a<b$ by definition. Note that $M(a)=U(a)=$ $\bigcap_{b^{\prime} \in \operatorname{Succ}(a)} \operatorname{Pred}\left(b^{\prime}\right)$ and $M(b)=\operatorname{Pred}(b)$. Since $a<b$ we find $\operatorname{Pred}(b)$ in the intersection defining $M(a)$. Hence, $M(a) \subseteq M(b)$ and $a<_{Q} b$.

$b_{1}, b_{2} \in B$ : We have $b_{1}<b_{2}$ in both $\operatorname{Stack}(P)$ and $Q$ if either $\operatorname{Pred}\left(b_{1}\right) \subset \operatorname{Pred}\left(b_{2}\right)$ or $\operatorname{Pred}\left(b_{1}\right)=\operatorname{Pred}\left(b_{2}\right)$ and $b_{1}$ precedes $b_{2}$ in $O$.

$a_{1}, a_{2} \in A$ : Recall from Lemma 2 of [FHM91] that $U(x) \subseteq U(y)$ iff $\operatorname{Succ}(x) \supseteq$ $\operatorname{Succ}(y)$. With this remark, the present case is dual to the preceding case. 
$a \| b$ in $P$ : Note that $a \notin M(b)=\operatorname{Pred}(b)$ but $a \in M(a)=U(a)$, therefore, if $a$ and $b$ are comparable in $Q$ then $b<_{Q} a$. The comparability $b<_{Q} a$ is in $Q$ exactly if all $a^{\prime} \in \operatorname{Pred}(b)$ are elements of $\operatorname{Pred}\left(b^{\prime}\right)$ for all $b^{\prime} \in \operatorname{Succ}(a)$. This, however, is the defining condition for $b<a$ in $\operatorname{Stack}(P)$.

The idea for our treatment of 3-interval irreducible posets will be the following. Suppose that $P=\left(X,<_{P}\right)$ is 3-interval irreducible, then, by Theorem $1 \operatorname{dim} B(P)=3$. Hence, $B(P)=(Y,<)$ contains some poset $Q$ from the list of 3 -irreducible orders. Assuming that $B(P)$ contains a specified 3-irreducible poset $Q$, we then derive informations about the functions $L: X \rightarrow Y$ and $U: X \rightarrow Y$. Since these two functions define an interval representation of $P$ on $B(P)$, information about $L$ and $U$ translates back to information about $P$.

This vague outline may motivate the study of properties that have to be required for two functions $L: X \rightarrow Y$ and $U: X \rightarrow Y$ from a set $X$ to a closed order $Q=\left(Y,<_{Q}\right)$, such that, there is a poset $P$ on $X$ with $B(P)=Q$ and $L$ and $U$ as given. First recall that

(1) $L(x)<_{Q} U(x)$ for all $x \in X$,

(2) $Y=\operatorname{Im}(L) \cup \operatorname{Im}(U)$.

With the next lemma we give a less trivial property.

Lemma 2. Let $y_{1}, y_{2}$ are incomparable elements of $Q=B(P)$

(1) if Pred $\left(y_{1}\right) \subseteq \operatorname{Pred}\left(y_{2}\right)$ then $y_{1} \in U(X)$,

(2) if $\operatorname{Succ}\left(y_{1}\right) \subseteq \operatorname{Succ}\left(y_{2}\right)$, then $y_{1} \in L(X)$.

Proof. This is an immediate consequence of the next Theorem.

Theorem 3. Let $P=\left(X,<_{P}\right)$ and $B(P)=\left(Y,<_{Q}\right)$. The following statements are equivalent:

(1) $y_{1} \not \leq y_{2}$ in $B(P)$

(2) there is an element $x \in X$ with $U(x) \leq y_{1}$ but $U(x) \not y_{2}$

(3) there is an element $x \in X$ with $L(x) \geq y_{2}$ but $L(x) \nsupseteq y_{1}$

Proof. The equivalence of (1) and (2) is an easy consequence of the following

$$
y=\{x \in X: U(x) \subseteq y\} \text {, for all elements } y \text { of } B(P) .
$$

First, suppose that $y=L\left(x_{0}\right)$ for some $x_{0} \in X$. If $x \in y$, i.e., $x \in L\left(x_{0}\right)=$ $\operatorname{Pred}\left(x_{0}\right)$, then $x<x_{0}$. From $x_{0} \in \operatorname{Succ}(x)$ it follows, that $U(x)=\bigcap_{x^{\prime} \in \operatorname{Succ}(x)} \operatorname{Pred}\left(x^{\prime}\right) \subseteq$ $\operatorname{Pred}\left(x_{0}\right)=y$. Conversely, let $U(x) \subseteq y$. From $x \in U(x)$ we obtain $x \in y$.

Now suppose that $y=U\left(x_{0}\right)$ for some $x_{0} \in X$. If $x \in y$, i.e., $x \in U\left(x_{0}\right)=$ $\bigcap_{x^{\prime} \in \operatorname{Succ}\left(x_{0}\right)} \operatorname{Pred}\left(x^{\prime}\right)$, then $x<x^{\prime}$ for all $x^{\prime}>x_{0}$. Therefore, $\operatorname{Succ}(x) \supseteq \operatorname{Succ}\left(x_{0}\right)$ and hence $U(x) \subseteq U\left(x_{0}\right)=y$. Conversely, let $U(x) \subseteq y$. From $x \in U(x)$ we again obtain $x \in y$. 
The equivalence of (1) and (3) is obtained by duality. The argument is based on the following observation: There is an isomorphism between $B\left(P^{d}\right)$ and $B(P)^{d}$, such that, the interval representation of $P^{d}$ on $B(P)^{d}$ is obtained from the interval representation of $P$ on $B(P)$ by interchanging $L$ and $U$.

Remark. Let an interval representation $L, U: P \rightarrow Q$ of $P$ on $Q$ be called a proper interval representation if for all $y_{1}, y_{2} \in \operatorname{Im}(L) \cup \operatorname{Im}(U)$ the 3 statements of Theorem 3 are equivalent. If $L, U: P \rightarrow Q$ is a proper interval representation, then the ordering of $Q$ restricted to $\operatorname{Im}(L) \cup \operatorname{Im}(U)$ is isomorphic to $B(P)$ and the interval representations of $P$ on the restriction of $Q$ and on $B(P)$ are equal with respect to this isomorphism. Hence, $B(P)$ can be characterized as the unique minimal poset which admits a proper interval representation of $P$.

1.1. Bipartite 3-Interval-Irreducible Orders. We are ready to show, how the list of bipartite 3-interval-irreducible orders given by [Tr81], see also [TrMo76], can be predicted. Although this list is known for more than 15 years, we think, that the method used here is new and interesting.

We start introducing some notation.

Definition 4. Let $P=\left(X,<_{P}\right)$ be a poset and $B(P)=(Y,<)$. An element $y \in Y$ is called low-made if $y=L(x)$ for some $x \in X$, it is called up-made if $y=U(x)$ for some $x \in X$.

Let $Q=(Y,<)$ be a poset. Label an element $y \in Y$ with $\ell$ if there is a $y^{\prime} \in Y$ such that $y \| y^{\prime}$, i.e., $y$ and $y^{\prime}$ are incomparable, and $\operatorname{Succ}(y) \subseteq \operatorname{Succ}\left(y^{\prime}\right)$ An element $y \in Y$ is labeled with $u$ if there is a $y^{\prime} \in Y$ such that $y \| y^{\prime}$ and $\operatorname{Pred}(y) \subseteq \operatorname{Pred}\left(y^{\prime}\right)$. This gives the $(\ell, u)$-labeling of $Q=(Y,<)$. Note that $y \in Y$ is labeled $\ell / u$ in this labeling, if $y$ is forced, by Lemma 2, to be low-made/up-made, whenever $Q=B(P)$ for some $P$

Now suppose, that $Q=(Y,<)$ is 3 -irreducible. Recall that a critical pair is a pair $\left(y_{1}, y_{2}\right)$ with $\operatorname{Pred}\left(y_{1}\right) \supseteq \operatorname{Pred}\left(y_{2}\right)$ and $\operatorname{Succ}\left(y_{1}\right) \subseteq \operatorname{Succ}\left(y_{2}\right)$. It is easy to see $(\mathrm{cf}$. [KeTr82, Tr91]), that the 3-irreducibility of $Q$ implies that every $y \in Y$ is contained in some critical pair. Hence, every element $y \in Y$ will receive at least one label in the $(\ell, u)$-labeling of $Q$. (See Figure 1 for the $(\ell, u)$-labelings of the 3-irreducible posets).

If every element of a poset $Q=(Y,<)$ has received a label in the $(\ell, u)$-labeling, then a minimal bipartite order $P$ with $B(P)=\widehat{Q}$ is easily obtained: For every $u$-labeled element $y \in Y$ take a minimal element $y^{u}$ and for every $\ell$-labeled element $y \in Y$ take a maximal element $y^{\ell}$. The relations are given by $y^{u}<z^{\ell}$ if and only if $y \leq z$ in $Q$ (for an example see Figure 2).

If $Q$ is 3-irreducible then, in view of the preceeding remarks, the order $P$ obtained by this process may be considerd as a candidate for the list of bipartite 3-intervalirreducible posets. To check the irreducibility we construct $B\left(P_{x}\right)$ for every point 


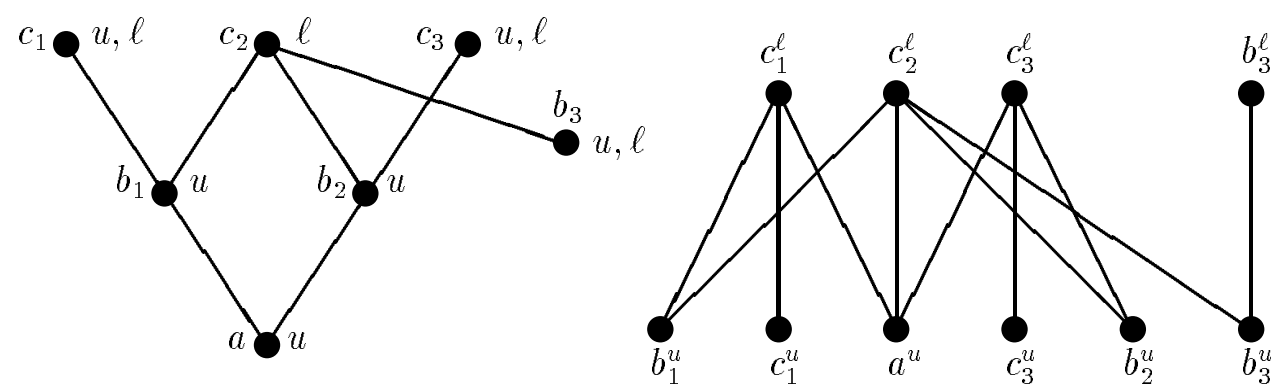

Figure 2. The 3 irreducible order $\mathrm{C}$ and the bipartite order $\mathcal{O}_{2}$ corresponding to the $(\ell, u)$-labeling of $\mathbf{C}$.

deleted suborder $P_{x}$ of $P$ and compute $\operatorname{dim} B\left(P_{x}\right)$. If $\operatorname{dim} B\left(P_{x}\right)=2$ for all $x$, then $P$ is indeed a 3 -interval-irreducible poset. The results are given in the list below.

Our notation and labelling for the collection of all 3 irreducible orders (see Figure 1) was introduced by Kelly [Ke77], it is also used in [Tr81] and [Tr91]. For bipartite 3interval irreducible posets we use the notation introduced in [TrMo76] (see also [Tr81]).

$Q=\mathbf{A}_{n}$ : Here we obtain the family $\mathcal{A}_{n}$.

$Q=\mathrm{B} \quad$ : Here we obtain $\mathcal{O}_{1}$.

$Q=\mathbf{C} \quad$ : Here we obtain $\mathcal{O}_{2}$.

$Q=\mathbf{D} \quad$ : Here we obtain $\mathcal{I}_{0}$.

$Q=\mathbf{E X}_{2}$ : Here we obtain $\mathcal{O}_{3}$.

$Q=\mathrm{CX}_{1}$ : The order $P$ obtained here is not irreducible.

Removing $b_{1}^{u}$ from $P$ we have $B\left(P_{b_{1}^{u}}\right)$ is the dual $\widehat{\mathbf{C}}^{d}$ of $\widehat{\mathbf{C}}$, i.e., $\operatorname{Idim}\left(P_{b_{1}^{u}}\right)=3$.

$Q=\mathrm{CX}_{2}$ : The order $P$ obtained here is not irreducible.

Removing $b_{1}^{u}$ and $b_{3}^{u}$ from $P$ we have $B\left(P_{b_{1}^{u}, b_{3}^{u}}\right)=\widehat{\mathbf{C}}^{d}$.

$Q=\mathrm{CX}_{3}$ : The order $P$ obtained here is not irreducible.

Removing $b_{1}^{u}$ and $a_{1}^{\ell}$ from $P$ we have $B\left(P_{b_{1}^{u}, a_{1}^{\ell}}\right)=\widehat{\mathbf{D}}$.

$Q=\mathbf{E X}_{1}$ : The order $P$ obtained here is not irreducible.

Removing $b_{2}^{u}$ from $P$ we have $B\left(P_{b_{2}^{u}}\right)=\widehat{\mathbf{E}_{0}}$.

$Q=\mathbf{F X}_{1}$ : The order $P$ obtained here is not irreducible.

Removing $\boldsymbol{a}_{1}^{\ell}$ from $P$ we have $B\left(P_{a_{1}^{\ell}}\right)=\widehat{\mathbf{F}_{0}}$.

$Q=\mathbf{F X}_{2}$ : The order $P$ obtained here is not irreducible.

Removing $a_{1}^{\ell}$ and $b_{3}^{u}$ from $P$ we have $B\left(P_{a_{1}^{\ell}, b_{3}^{u}}\right)=\widehat{\mathbf{F}_{0}}$.

$Q=\mathbf{E}_{n}$ : Here we obtain the family $\mathcal{E}_{n}$.

$Q=\mathbf{F}_{n}$ : Here we obtain the family $\mathcal{F}_{n}$.

$Q=\mathbf{I}_{n} \quad$ : Here we obtain the family $\mathcal{I}_{n+1}$.

$Q=\mathbf{G}_{n}$ : Here we obtain the family $\mathcal{G}_{n}$.

$Q=\mathbf{H}_{n}$ : Here we obtain the family $\mathcal{H}_{n}$.

$Q=\mathbf{J}_{n}$ : The order $P$ obtained here is not irreducible.

Removing $c^{\ell}$ and $d^{u}$ from $P$ we have $B\left(P_{c^{\ell}, d^{u}}\right)=\widehat{\mathbf{G}_{n}}$. 
Let $Q=(Y,<)$ be a 3 -irreducible poset and let $P$ be the bipartite order corresponding to the $(\ell, u)$-labeling of $Q$. A label $\rho \in\{\ell, u\}$ at an element $y \in Y$ is called essential if $\operatorname{dim} B\left(P_{y^{\circ}}\right)=2$. Note that, $P$ is 3-interval irreducible exactly if every label of $Q$ is essential.

Above we have compiled a list of bipartite 3 -interval-irreducible posets. To prove that this list is indeed complete, i.e., consists of all bipartite 3-interval-irreducible posets, however, a large amount of work remains. This work has been accomplished by Trotter [Tr81]. In the next section we give a modified version of his argument to prove our Theorem 4.

Remark. The split of a poset $P=(X,<)$ is the bipartite poset with maximal elements $\left\{x^{\ell}: x \in X\right\}$ and minimal elements $\left\{x^{u}: x \in X\right\}$ with $x^{u}<y^{\ell}$ in $\operatorname{Split}(P)$ iff $x \leq y$ in $P$. Note that, as a consequence of Trotter's result we can state that every bipartite 3 -interval irreducible poset is a subposet of the split of some 3-irreducible poset.

\section{General 3-Interval-Irreducible Posets}

The program for dealing with the general (non-bipartite) case is the following: In the first part, we use the techniques developed in [Tr81] for the characterization the bipartite 3-interval irreducible orders to show that every 3-interval irreducible order contains a partial stack or a reduced partial stack of a bipartite 3-interval irreducible order. In the second part, we then attempt to characterize the 3 -interval irreducible orders among the candidate posets obtained in the first part.

\subsection{Partial Stacks and Reduced Partial Stacks.}

Definition 5. Let $P=\left(X,<_{P}\right)$ be a (connected) bipartite poset with minimal elements $X_{1}$ and maximal elements $X_{2}$. A partial stack of $P$ is an order $Q=\left(X,<_{Q}\right)$ such that for all $x_{1} \in X_{1}$ and $x_{2} \in X_{2}$ we have $x_{1}<_{P} x_{2}$ exactly if $x_{1}<_{Q} x_{2}$.

A partial stack $Q$ of $P$ may contain parallel elements. The reduced partial stack corresponding to $Q$ is obtained by contracting each set of pairwise parallel elements to a single point.

Remark. Let $P=\left(X,<_{P}\right)$ be a (connected) bipartite poset with minimal elements $X_{1}$ and maximal elements $X_{2}$. Let $\mathbb{E}_{P}$ be the set of all extensions $Q$ of $P$ satisfying $x_{1}<_{Q} x_{2}$ iff $x_{1}<_{P} x_{2}$, i.e., $\mathbb{E}_{P}$ is the set of all partial stacks of $P$. Define a poset on $\mathbb{E}_{P}$ by $Q_{1} \leq Q_{2}$ if $Q_{2}$ is an extension of $Q_{1}$. If $Q$ is any maximal element of this poset, then $Q$ is isomorphic to $\operatorname{Stack}(P)$.

The main theorem of the present section is.

Theorem 4. If $P$ is a 3-interval irreducible poset then $P$ contains a reduced partial stack of some bipartite 3-interval irreducible poset. 
To prove the theorem we go along Trotter's, 'Stacks and Splits' [Tr81] and rewrite the important lemmas and theorems. We omit the assumption that $P$ is a height one poset, we replace $\operatorname{Stack}(P)$ by $B(P)$ and instead of dealing with elements in Stack $(P)$ corresponding to maximal or minimal elements in $P$ we deal with low-made and upmade elements of $B(P)$. As a first example note that Lemma 3 implies an analogue of Trotter's Lemma 4.

Theorem 5 (Trotter's Theorem 6). If $P$ is a poset and $B(P)$ contains a crown $\boldsymbol{A}_{n}$ for some $n \geq 0$, then there exists an integer $m$ with $0 \leq m \leq n$ so that $P$ contains $\boldsymbol{A}_{m}$.

Proof. Choose the smallest integer $k \geq 0$ for which $B(P)$ contains $\mathbf{A}_{k}$. We will then show that $P$ contains $\mathbf{A}_{k}$.

Of all copies of $\mathbf{A}_{k}$ contained in $B(P)$, choose one for which the integer $t=\mid\left\{b_{i}\right.$ : $1 \leq i \leq k+3, b_{i}$ is low-made $\}|+|\left\{a_{i}: 1 \leq i \leq k+3, a_{i}\right.$ is up-made $\} \mid$ is as large as possible. If $t=2 k+6$, then for $0 \leq i \leq k+3$ there are elements $a_{i}^{\prime}, b_{i}^{\prime}$ in $P$ with $L\left(b_{i}^{\prime}\right)=b_{i}$ and $U\left(a_{i}^{\prime}\right)=a_{i}$. These elements form a copy of $\mathbf{A}_{k}$ in $P$.

Assume that $t<2 k+6$ and without loss of generality let $b_{k+2}$ be a non low-made element. Since $a_{1} \| b_{k+2}$ in $B(P)$, it follows from Theorem 3 , that there is an element $x \in X$ so that $L(x) \geq b_{k+2}$ and $L(x) \| a_{1}$. If $L(x) \| a_{i}$ for each $i=2,3, \ldots, k+1$, then $x$ can replace $b_{k+2}$ in this copy of $\mathbf{A}_{k}$. So we must have $L(x) \geq a_{i}$ for some $i$ with $2 \leq i \leq k+1$. Let $i_{0}$ be the smallest such integer. Then it follows that the subposet of $B(P)$ generated by $\left\{a_{1}, a_{2}, \ldots, a_{i_{0}}, a_{k+3}\right\} \cup\left\{b_{1}, b_{2}, \ldots, b_{i_{0}-1}, L(x), b_{k+3}\right\}$ is $\mathbf{A}_{i_{0}-2}$. But $i_{0}-2<k$, this is a contradiction.

Theorem 6 (Trotter's Theorem 7). Let $P$ be a poset and suppose that $P$ does not contain a crown $\boldsymbol{A}_{n}$ for any $n \geq 0$. If $y$ is an up-made (low-made) element of $B(P)$ and $F$ is a connected subposet of Inc(y), then there exists a low-made (up-made) element $x$ in $B(P)$ so that $x \geq y(y \geq x)$ and $F \subseteq \operatorname{Inc}(x)$.

Proof. We prove the theorem when $y$ is up-made. If $y$ is also low-made, then $x=y$ and we are done.

Suppose that $y$ is not low-made. Let $y^{\prime}>y$ be low-made and note that the existence of a low-made element $y^{\prime \prime}>y$ with $y^{\prime \prime}|| y^{\prime}$ is guaranteed by Theorem 3 . Therefore, there are at least two incomparable low-made elements in $\operatorname{Succ}_{B(P)}(y)$

If there is a $z \in F$ with $x \geq z$ for all low-made elements $x>y$, then $y \geq z$ by Theorem 3. A contradiction.

We therefore may assume, that there are elements $x, x^{\prime} \in \operatorname{Succ}_{B(P)}(y)$ and $z, z^{\prime} \in F$ such that $z<x$ and $z^{\prime}<x^{\prime}$ but $x \| z^{\prime}$ and $x^{\prime} \| z$. Now let $a_{1}, \ldots, a_{n}$ be a fence in $F$ from $z^{\prime}$ to $z$. It follows that $\left\{x, y, x^{\prime}, a_{1}, \ldots, a_{n}\right\}$ contains a crown. A contradiction. 
Let $P=\left(X,<_{P}\right)$ be a 3 -interval irreducible poset. Since the dimension of $B(P)$ is $3, B(P)$ contains a 3 -irreducible poset. Suppose first, that $B(P)$ contains a crown $\mathbf{A}_{n}$ for some $n \geq 0$. Then, it follows from Theorem 5, that $P$ also contains a crown $\mathbf{A}_{m}$ for some $0 \leq m \leq n$. Since $P$ is irreducible, we conclude, that $P=\mathbf{A}_{n}$. We may, therefore, assume in the remainder of the argument, that $B(P)$ does not contain a crown $\mathbf{A}_{n}$. The remainder of the argument is divided into a sequence of cases.

When discussing the case of a 3 -irreducible order $Q=\left(Y,<_{Q}\right)$ contained in $B(P)$, we assume, that a copy of $Q$ in $B(P)$ has been chosen. We refer to the elements of this copy of $Q$ via the labeling of [Ke77] and [Tr81], see Figure 1. If $y$ is an element of $Q$, then we say there is an element $y^{\ell}$ in $P$ if we are sure about the existence of a low-made element $y^{\prime} \geq y$ with $\operatorname{Pred}_{B(P)}\left[y^{\prime}\right] \cap Y=\operatorname{Pred}_{Q}[y]$. The element $y^{\ell}$ then is any preimage of $y^{\prime}$ under $L$, i.e., $L\left(y^{\ell}\right)=y^{\prime}$. Dually, we say there is an element $y^{u}$ in $P$ if we are sure about the existence of an up-made element $y^{\prime \prime} \leq y$ with $\operatorname{Succ}_{B(P)}\left[y^{\prime \prime}\right] \cap Y=\operatorname{Succ}_{Q}[y]$. The element $y^{\ell}$ then is any element with $U\left(y^{u}\right)=y^{\prime \prime}$.

We will make extensive use of the following easy consequence of Theorem 6 .

Lemma 3. Let $P=\left(X,<_{P}\right)$ be a crown-free poset and suppose that a 3-irreducible poset $Q=\left(Y,<_{Q}\right)$ is contained in $B(P)$. If $y$ is an element of $Q$ such that Inc $c_{Q}(y)$ is connected and for all $y^{\prime}$ in $Q$ with $y^{\prime} \notin \operatorname{Pred}_{Q}[y]\left(y^{\prime} \notin \operatorname{Succ}_{Q}[y]\right)$ there is a $y^{\prime \prime} \in \operatorname{Inc}_{Q}(y)$ with $y^{\prime} \geq y^{\prime \prime}\left(y^{\prime} \leq y^{\prime \prime}\right)$, then there is an element $y^{\ell}\left(y^{u}\right)$ in $P$.

In each case we choose a particular 3 -irreducible poset $Q=\left(Y,<_{Q}\right)$ contained in $B(P)$ and suppose that $B(P)$ does not contain the 3 -irreducible posets treated in the previous cases. These assumptions together with Theorem 3, Theorem 6 and Lemma 3 will allow us to show that there is an element $y^{\ell}\left(y^{u}\right)$ for every essential $\ell$-labeled (u-labeled) element $y$ of $Q$. Let $X^{u} \subseteq X$ be the set of these up-made elements and $X^{\ell} \subseteq X$ be the set of these low-made elements. The bipartite order $\left(X^{u} \times X^{\ell}\right) \cap<_{P}$ is isomorphic to a bipartite 3 -interval irreducible poset $R$. Therefore, $P$ contains a partial stack of $R$.

Case 1. $B(P)$ contains D.

We apply Lemma 3 to obtain elements $b_{1}^{\ell}, b_{2}^{\ell}, b_{3}^{\ell}, c_{1}^{\ell}, c_{2}^{\ell}$ and $a^{u}, b_{1}^{u}, b_{2}^{u}, b_{3}^{u}$ in $P$. The bipartite order $\left(X^{u} \times X^{\ell}\right) \cap<_{P}$ forms a copy of $\mathcal{I}_{0}$ in $P$. Hence, $P$ contains a partial stack of $\mathcal{I}_{0}$.

Case 2. $B(P)$ contains $\mathbf{C}$.

From Lemma 3 we may assume, that there are elements $c_{1}^{\ell}, c_{3}^{\ell}, b_{3}^{\ell}$ and $a^{u}, b_{3}^{u}, c_{1}^{u}, c_{3}^{u}$.

Suppose, that there is no element $c_{2}^{\ell}$, then by Theorem 6 , there are elements $c^{\prime}>c_{2}$ and $c^{\prime \prime}>c_{2}$ in $B(P)$ with $c_{1} \in \operatorname{Inc}\left(c^{\prime \prime}\right)$ but $c^{\prime \prime}>c_{3}$ and $c_{3} \in \operatorname{Inc}\left(c^{\prime}\right)$ but $c^{\prime}>c_{1}$. This implies, that $\left\{a, c_{1}, c^{\prime \prime}, b_{3}, c^{\prime}, c_{3}\right\}$ form a copy of $\mathbf{D}$ in $B(P)$. The contradiction allows us to assume an element $c_{2}^{\ell}$. 
Assume, that $b_{1}$ is low-made, then choose an up-made element $b_{1}^{\prime}<b_{1}$ with $b_{2}, c_{3} \subseteq$ Inc $\left(b_{1}^{\prime}\right)$. If $b_{1}^{\prime}<b_{3}$, then $\left\{c_{2}, b_{2}, a, c_{1}, b_{1}^{\prime}, b_{3}\right\}$ form a copy of $\mathbf{D}^{d}$ in $B(P)$. We conclude, that $b_{3} \in \operatorname{Inc}\left(b_{1}^{\prime}\right)$, hence, any $U$-preimage of $b_{1}^{\prime}$ is $b_{1}^{u}$. Symmetrically, we may assume an element $b_{2}^{u}$. The bipartite order with maximal elements $c_{1}^{\ell}, c_{2}^{\ell}, c_{3}^{\ell}, b_{3}^{\ell}$ and minimal elements $a^{u}, b_{1}^{u}, b_{2}^{u}, b_{3}^{u}, c_{1}^{u}, c_{3}^{u}$ forms a copy of $\mathcal{O}_{2}$ in $P$. Hence, $P$ contains a partial stack of $\mathcal{O}_{2}$.

Case 3. $B(P)$ contains $\mathbf{C X}_{3}$.

From Lemma 3 we may assume that there are elements $a_{3}^{\ell}, b_{1}^{\ell}, b_{3}^{\ell}, c^{\ell}$ and $a_{1}^{u}, a_{3}^{u}, b_{3}^{u}$. Choose a low-made element $b_{2}^{\prime}>b_{2}$ with $b_{3}, c \in \operatorname{Inc}\left(b_{2}^{\prime}\right)$. If $b_{2}^{\prime}>b_{1}$, then $\left\{a_{2}, b_{1}, b_{2}^{\prime}, a_{3}, c, b_{3}\right\}$ form a copy of $\mathbf{D}$ in $B(P)$. Therefore, we may assume an element $b_{2}^{\ell}$ in $P$.

If there is no element $a_{2}^{u}$, then there are up-made elements $a_{2}^{\prime}<a_{2}$ and $a_{2}^{\prime \prime}<a_{2}$ with $a_{1} \| a_{2}^{\prime}$ but $a_{2}^{\prime}<a_{3}$ and $a_{3} \| a_{2}^{\prime \prime}$ but $a_{2}^{\prime \prime}<a_{1}$. However, it follows, that $\left\{b_{2}, a_{1}, a_{2}^{\prime \prime}, b_{3}, a_{2}^{\prime}, a_{3}\right\}$ form a copy of $\mathbf{D}^{d}$. The contradiction shows that we may assume an element $a_{2}^{u}$.

The bipartite order corresponding to these elements $y^{\ell}$ and $y^{u}$ as maximals and minimals forms a copy of $\mathcal{I}_{0}$ in $P$.

Case 4. $B(P)$ contains $\mathbf{C X}_{2}$.

From Lemma 3 we obtain elements $a_{1}^{\ell}, a_{3}^{\ell}, c^{\ell}$ and $a_{1}^{u}, a_{3}^{u}, c^{u}$.

The same argument used in Case 3 , allows us to assume an up-made element $a_{2}^{u}$. Next, choose a low-made element $b_{3}^{\prime} \geq b_{3}$ with $\left\{b_{1}, b_{2}, a_{1}\right\} \in \operatorname{Inc}\left(b_{3}^{\prime}\right)$. If $b_{3}^{\prime}>c$, then $B(P)$ contains $\mathbf{C X}_{3}$. Therefore, we assume an element $b_{3}^{\ell}$. By symmetry, we may also assume an element $b_{1}^{u}$. Finally, choose a low-made element $b_{2}^{\prime}>b_{2}$ with $c \in \operatorname{Inc}\left(b_{2}^{\prime}\right)$. Obviously, $b_{1} \nless b_{2}^{\prime}$ and $c_{2} \nless b_{2}^{\prime}$. Let $x$ be a preimage of $b_{2}^{\prime}$ under $L$. The bipartite order with maximal elements $a_{1}^{\ell}, a_{3}^{\ell}, b_{1}^{\ell}, x, b_{3}^{\ell}, c^{\ell}$ and minimal elements $a_{1}^{u}, a_{2}^{u}, a_{3}^{u}, c^{u}$ forms a copy of $\mathcal{O}_{2}^{d}$ in $P$.

Case 5. $B(P)$ contains $\mathbf{C X}_{1}$.

If $b_{1}$ is not low-made, then choose a low-made element $b_{1}^{\prime}>b_{1}$ with $\left\{a_{3}, b_{2}, c\right\} \subseteq \operatorname{Inc}\left(b_{1}^{\prime}\right)$. If $b_{1}^{\prime}>b_{3}$, then $\left\{a_{2}, b_{3}, b_{1}^{\prime}, a_{1}, c, b_{2}\right\}$ form a copy of $\mathbf{D}$. Therefore, we may assume that there is an element $b_{1}^{\ell}$ in $P$.

If there is no element $a_{2}^{u}$, then there are up-made elements $a_{2}^{\prime}<a_{2}$ and $a_{2}^{\prime \prime}<a_{2}$ with $a_{1} \| a_{2}^{\prime}$ but $a_{2}^{\prime}<a_{3}$ and $a_{3} \| a_{2}^{\prime \prime}$ but $a_{2}^{\prime \prime}<a_{1}$. It follows, that $\left\{c, a_{1}, a_{2}^{\prime \prime}, b_{3}, a_{2}^{\prime}, a_{3}\right\}$ form a copy of $\mathbf{D}^{d}$. The contradiction shows, that we may assume an element $a_{2}^{u}$ in $P$.

If $b_{2}$ is not low-made, then choose a low-made element $b_{2}^{\prime}>b_{2}$ with $a_{1}, b_{1} \subset \operatorname{Inc}\left(b_{2}^{\prime}\right)$. If $b_{2}^{\prime}>b_{3}$, then $\left\{a_{1}, a_{2}, a_{3}, b_{1}, c, b_{3}, b_{2}^{\prime}\right\}$ form a copy of $\mathbf{C X}_{3}$. We, therefore, assume an element $b_{2}^{\ell}$ in $P$.

From Lemma 3 we obtain elements $a_{1}^{\ell}, a_{3}^{\ell}, b_{3}^{\ell}$ and $a_{1}^{u}, a_{3}^{u}, b_{3}^{u}$. We also may choose a low-made element $c^{\prime}>c$ with $b_{3} \in \operatorname{Inc}\left(c^{\prime}\right)$. Let $x$ be a preimage of $c^{\prime}$ under $L$. 
The bipartite order with maximal elements $a_{1}^{\ell}, a_{3}^{\ell}, b_{1}^{\ell}, b_{2}^{\ell}, b_{3}^{\ell}, x$ and minimal elements $a_{1}^{u}, a_{2}^{u}, a_{3}^{u}, b_{3}^{u}$ forms a copy of $\mathcal{O}_{2}^{d}$ in $P$.

Case 6. $B(P)$ contains $\mathbf{E X}_{2}$.

Suppose, that $b_{2}$ is not low-made and there is no low-made element $b_{2}^{\prime}>b_{2}$ with $c, b_{3} \in \operatorname{Inc}\left(b_{2}^{\prime}\right)$. Then it follows, that there exist points $b_{4}, b_{5}>b_{2}$ with $b_{3} \in \operatorname{Inc}\left(b_{4}\right)$ and $c, b_{1} \in \operatorname{In} c\left(b_{5}\right)$. This implies, that $b_{5}>b_{3}$. If $b_{4}>c$, then $\left\{a_{2}, b_{1}, c, a_{3}, b_{5}, b_{3}\right\}$ generate a copy of $\mathbf{D}$. So we must have $b_{4} \| c$, and thus $b_{4}>b_{1}$. However, this implies that $\left\{c, b_{1}, b_{4}, b_{2}, b_{3}, a_{2}, a_{3}\right\}$ generate a copy of $\mathbf{C X}_{1}$ in $B(P)$. The contradiction allows us to assume an element $b_{2}^{\ell}$ in $P$. Since $\mathbf{E X}_{2}=\mathbf{E X}_{2}^{d}$ we obtain $a_{2}^{u}$ from the dual argument.

If $c$ is not low-made, then choose an element $c^{\prime}>c$ with $a_{3}, b_{2} \in \operatorname{Inc}\left(c^{\prime}\right)$. If $c^{\prime}>b_{3}$, then $\left\{c^{\prime}, b_{3}, a_{2}, b_{2}, a_{1}, b_{1}\right\}$ form a copy of $\mathbf{D}^{d}$. We, therefore, may assume an element $c^{\ell}$. Dually, we may assume $a_{1}^{u}$.

From Lemma 3 we obtain elements $a_{3}^{\ell}, b_{1}^{\ell}, b_{3}^{\ell}$ and $a_{3}^{u}, b_{1}^{u}, b_{3}^{u}$. The bipartite order with maximal elements $a_{3}^{\ell}, b_{1}^{\ell}, b_{2}^{\ell}, b_{3}^{\ell}, c^{\ell}$ and minimal elements $a_{1}^{u}, a_{2}^{u}, a_{3}^{u}, b_{1}^{u}$ forms a copy of $\mathcal{O}_{3}$ in $P$.

Case 7. $B(P)$ contains $\mathbf{F X}_{1}$.

If $b_{2}$ is not low-made, then choose a low-made element $b_{2}^{\prime}>b_{2}$ with $b_{2}^{\prime} \| b_{3}$. If $b_{2}^{\prime}>b_{1}$, then $B(P)$ contains $\mathbf{D}$. We, hence, assume an element $b_{2}^{\ell}$ in $P$.

If there is no element $a_{1}^{u}$, then there are up-made elements $a_{1}^{\prime}<a_{1}$ and $a_{1}^{\prime \prime}<a_{1}$ with $a_{2}, b_{3} \in \operatorname{Inc}\left(a_{1}^{\prime}\right)$ but $a_{1}^{\prime}<a_{3}$ and $a_{3} \in \operatorname{Inc}\left(a_{1}^{\prime \prime}\right)$ but $a_{1}^{\prime \prime}<b_{3}$. It follows, that $\left\{c, b_{3}, a_{1}^{\prime \prime}, b_{1}, a_{1}^{\prime}, a_{3}\right\}$ form a copy of $\mathbf{D}^{d}$. The contradiction shows, that we may assume an element $a_{1}^{u}$ in $P$.

If there is no element $a_{2}^{u}$, then there are up-made elements $a_{2}^{\prime}<a_{2}$ and $a_{2}^{\prime \prime}<a_{2}$ with $a_{1} \| a_{2}^{\prime}$ but $a_{2}^{\prime}<a_{3}$ and $a_{3} \| a_{2}^{\prime \prime}$ but $a_{2}^{\prime \prime}<a_{1}$. It follows, that $\left\{b_{2}, a_{1}, a_{2}^{\prime \prime}, b_{3}, a_{2}^{\prime}, a_{3}\right\}$ form a copy of $\mathbf{D}^{d}$. The contradiction shows, that we may assume an element $a_{2}^{u}$.

From Lemma 3 we obtain low-made elements $a_{3}^{\ell}, b_{1}^{\ell}, b_{3}^{\ell}, c^{\ell}$ and up-made elements $a_{3}^{u}, b_{1}^{u}, b_{3}^{u}$. The bipartite order with maximal elements $a_{3}^{\ell}, b_{1}^{\ell}, b_{2}^{\ell}, b_{3}^{\ell}, c^{\ell}$ and minimal elements $a_{1}^{u}, a_{2}^{u}, a_{3}^{u}, b_{1}^{u}, b_{3}^{u}$ forms a copy of $\mathcal{F}_{0}$ in $P$.

Case 8. $B(P)$ contains $\mathbf{E X}_{1}$.

Choose a low-made element $b_{2}^{\prime}>b_{2}$ with $a_{3}, b_{3} \in \operatorname{Inc}\left(b_{2}^{\prime}\right)$. If $b_{2}^{\prime}>b_{1}$ and $b_{2}^{\prime}>b_{4}$, then $\left\{b_{2}^{\prime}, b_{1}, a_{1}, b_{3}, a_{2}, b_{4}\right\}$ generate $\mathbf{D}^{d}$. If $b_{2}^{\prime}>b_{1}$ and $b_{2}^{\prime} \| b_{4}$, then replacing $b_{2}$ by $b_{2}^{\prime}$ we obtain $\mathbf{E X}_{2}$. If $b_{2}^{\prime}>b_{4}$ and $b_{2}^{\prime}|| b_{1}$, then the same elements also generate $\mathbf{E X}_{2}$. Hence, we may assume an element $b_{2}^{\ell}$ in $P$.

Choose a low-made element $b_{3}^{\prime}>b_{3}$, such that $b_{2} \in \operatorname{Inc}\left(b_{3}^{\prime}\right)$. If $b_{3}^{\prime}>b_{1}$, then $\left\{a_{1}, a_{2}, a_{3}, b_{1}, b_{2}, b_{3}, b_{3}^{\prime}\right\}$ forms a copy of $\mathbf{F X}_{1}$ in $B(P)$. If $b_{3}^{\prime}>b_{4}$, then $\left\{a_{1}, a_{2}, a_{3}, b_{2}, b_{3}, b_{3}^{\prime}, b_{4}\right\}$ generates $\mathbf{F X}_{1}$. We may, therefore, assume that there is an element $b_{3}^{\ell}$ in $P$.

If there is no element $a_{1}^{u}$, then choose an up-made element $a_{1}^{\prime}<a_{1}$ with $a_{2}, b_{4} \in$ 
Inc $\left(a_{1}^{\prime}\right)$. If $a_{1}^{\prime}<a_{3}$, then $B(P)$ contains a copy of $\mathbf{F X}_{1}^{d}$. Thus, we may assume $a_{3} \| a_{1}^{\prime}$, i.e., we may assume an element $a_{1}^{u}$ in $P$. By symmetry, we may also assume an element $a_{2}^{u}$ in $P$.

From Lemma 3 we obtain elements $a_{3}^{\ell}, b_{1}^{\ell}, b_{4}^{\ell}$ and $a_{3}^{u}, b_{1}^{u}, b_{4}^{u}$. The bipartite order $\left(X^{u} \times\right.$ $\left.X^{\ell}\right) \cap<_{P}$ is a copy of $\mathcal{E}_{0}$ in $P$.

Case 9. $B(P)$ contains $\mathbf{F X}_{2}$.

If there is no low-made element $c^{\prime}>c$ with $b_{1}, b_{3} \in \operatorname{Inc}\left(c^{\prime}\right)$, then choose $c_{1}>c$ and $c_{2}>c$ with $b_{3} \| c_{1}$ but $b_{1}<c_{1}$ and $b_{1}{ }^{\prime} \| c_{2}$ but $b_{3}<c_{2}$. It follows, that $B(P)$ contains a copy of $\mathbf{D}$. We, therefore, may assume an element $c^{\ell}$. Dually, we also assume an element $a_{2}^{u}$ in $P$.

Now, choose a low-made element $b_{3}^{\prime} \geq b_{3}$ with $c, b_{2} \in \operatorname{Inc}\left(b_{3}^{\prime}\right)$. If $b_{3}^{\prime}>b_{1}$, then $B(P)$ contains D. We, therefore, assume an element $b_{3}^{\ell}$ in $P$. Dually, we also assume an element $a_{1}^{u}$.

From Lemma 3 we may assume elements $a_{3}^{\ell}, b_{1}^{\ell}, b_{2}^{\ell}$ and $a_{3}^{u}, b_{1}^{u}, b_{2}^{u}$ in $P$. The bipartite order generated by these elements is a copy of $\mathcal{F}_{0}$ in $P$.

In cases 10 to 13 the next lemma will find repeated applications.

Lemma 4. Let $P$ be a poset such that $B(P)$ contains no $\boldsymbol{D}, \boldsymbol{C X}_{3}$, nor their duals or a crown $\boldsymbol{A}_{n}$. Let $a_{1}, b_{1}, a_{2}, b_{2}, a_{3} \ldots, a_{n}, b_{n}$ be a fence in $B(P)$ and $x>b_{i}$ for some $i \in\{1, \ldots, n\}$.

(1) If $j_{1}$ is the minimal, $j_{2}$ the maximal integer with $x>a_{j_{1}}$ and $x>a_{j_{2}}$, then $x>a_{j}$ when $j_{1} \leq j \leq j_{2}$.

(2) If $j_{1}$ is the minimal, $j_{2}$ the maximal integer with $x>b_{j_{1}}$ and $x>b_{j_{2}}$, then $x>b_{j}$ when $j_{1} \leq j \leq j_{2}$.

Proof. If $x \| a_{j}$ for some $j_{1} \leq j \leq j_{2}$, then $B(P)$ contains a crown.

If $x \| b_{j}$ but $x>b_{j-1}$ and $x>b_{j+1}$, then $B(P)$ contains $\mathbf{D}^{d}$. Finally, suppose that $x>b_{j_{*}}$ and $x>b_{j^{*}}$ and $j_{*}+2<j^{*}$ but $x \| b_{j}$ for all $j_{*}<j<j^{*}$. Then from the first part we know that $x>a_{j_{*}+1}, a_{j_{*}+2}$ and the elements of $\left\{a_{j_{*}}, a_{j_{*}+1}, a_{j_{*}+2}, b_{j_{*}}, b_{j_{*}+1}, b_{j_{*}+2}, x\right\}$ generate a copy of $\mathrm{CX}_{3}$ in $B(P)$.

Note that the dual of this lemma is also true, i.e, if $x$ is below some element $a_{i}$, then the elements of the fence comparable with $x$ are intervals of $a$ 's and $b$ 's.

Case 10. $B(P)$ contains $\mathbf{F}_{n}$.

We also assume that $B(P)$ does not contain $\mathbf{F}_{m}, \mathbf{E}_{m}$ or $\mathbf{E}_{m}^{d}$ when $0 \leq m<n$.

Now suppose, that $2 \leq i \leq n+2$. Choose a low-made element $x$ with $x \geq b_{i}$ and $x \| d$.

Suppose, that $x<e$, it follows that $x \| b_{1}$. Let $j_{1}$ be the minimal, $j_{2}$ the maximal integer with $x>a_{j_{1}}$ and $x>a_{j_{2}}$. If $j_{2}=j_{1}+1$, then there is an element $b_{i}^{\ell}$ in 
$P$. Otherwise, $\left\{a_{1}, \ldots, a_{j_{1}}, a_{j_{2}}, \ldots, a_{n+2}\right\} \cup\left\{b_{1}, \ldots, b_{j_{1}}, x, b_{j_{2}+1}, \ldots, b_{n+2}\right\} \cup\{c, d, e\}$ generates $\mathbf{F}_{m}$ for some $m<n$.

Now suppose, that $x \| e$. If $x \| a_{n+2}$ then $\left\{a_{j_{2}}, \ldots, a_{n+2}\right\} \cup\left\{x, b_{j_{2}+1}, \ldots, b_{n+2}\right\} \cup$ $\{c, d, e\}$ generates $\mathbf{F}_{m}$ for some $m<n$. We, henceforth, may assume that $x>a_{n+2}$. If $x>b_{1}$, then $\left\{c, d, \epsilon, a_{n+2}, x, b_{1}\right\}$ form a copy of $\mathbf{D}$. Now assume, that $x \| b_{1}$ and $j_{1}>1$, then $\left\{a_{1}, \ldots, a_{j_{1}}\right\} \cup\left\{b_{1}, \ldots, b_{j_{1}}, x\right\} \cup\{d, \epsilon\}$ generate $\mathbf{E}_{m}$ for some $m<n$. Finally, if $b_{1}, e \in \operatorname{Inc}(x)$ and $x>a_{1}, x>a_{n+2}$, then $\left\{a_{1}, a_{n+2}, b_{1}, c, d, e, x\right\}$ form a copy of $\mathbf{F X}_{1}^{d}$. We may, therefore, assume an element $b_{i}^{\ell}$ in $P$.

By symmetry and duality we may also assume elements $a_{i}^{u}$, when $1 \leq i \leq n+1$. From Lemma 3 we obtain $a_{n+2}^{\ell}, b_{1}^{\ell}, e^{\ell}, d^{\ell}$ and $a_{n+2}^{u}, b_{1}^{u}, c^{u}, d^{u}$ in $P$. The bipartite order corresponding to these elements form a copy of $\mathcal{F}_{n}$ in $P$.

Case 11. $B(P)$ contains $\mathbf{E}_{n}$.

We also assume that $B(P)$ does not contain $\mathbf{F}_{m}, \mathbf{E}_{m}$ or $\mathbf{E}_{m}^{d}$ when $0 \leq m<n$.

Now suppose, that $1 \leq i \leq n+1$. Choose an up-made element $a^{\prime}$ with $a^{\prime} \leq a_{i}$ and $a^{\prime} \| b_{i+2}$. Assume, that $a^{\prime}<c$ and let $j_{0}$ be the minimal index $j$ with $a^{\prime}<a_{j}$. If $j_{0}>1$ then $\left\{a_{j_{0}-1}, a_{j_{0}}, \ldots, a_{i+1}\right\} \cup\left\{b_{j_{0}}, b_{j_{0}+1}, \ldots, b_{i+1}\right\} \cup\left\{a^{\prime}, c\right\}$ forms a copy of $\mathbf{E}_{m}^{d}$ where $m<n$. If $j_{0}=1$ then $\left\{a_{1}, a_{2}, \ldots, a_{i+1}\right\} \cup\left\{b_{1}, b_{2}, \ldots, b_{i+1}\right\} \cup\left\{a^{\prime}, c, d\right\}$ forms a copy of $\mathbf{F}_{m}^{d}$ where $m<n$. The contradiction allows us to conclude $a^{\prime} \| c$. Let $j_{1}\left(j_{2}\right)$ be the minimal (maximal) index $j$ with $a^{\prime}<b_{j}$. If $j_{2} \neq j_{1}+1$, then $\left\{a_{1}, \ldots, a_{j_{1}-1}, a^{\prime}, a_{j_{2}+1} \ldots, a_{n+2}\right\} \cup\left\{b_{1}, \ldots, b_{j_{1}}, b_{j_{2}}, \ldots, b_{n+3}\right\} \cup\{c, d\}$ forms a copy of $\mathbf{E}_{m}^{d}$ where $m<n$. We may, therefore, assume elements $a_{1}^{u}, \ldots, a_{n+1}^{u}$ and by symmetry also $a_{n+2}^{u}$.

Next choose an integer $i$ with $2 \leq i \leq n+2$ and a low-made element $x \geq b_{i}$ with $x \| c$. Suppose, that $x \| d$. If $x>a_{1}$ and $x>a_{n+2}$, then if $b_{1}, b_{n+3} \in \operatorname{Inc}(x)$, a copy of $\mathbf{E X}_{1}$ is generated by $\left\{b_{1}, x, b_{n+3}, d, a_{1}, a_{n+2}, \epsilon\right\}$. The same point set generates $\mathbf{E X}_{2}$ if only one of $b_{1}$ and $b_{n+3}$ is incomparable with $x$. It contains $\mathbf{D}^{d}$ if $b_{1}, b_{n+3}<x$. We now assume, without loss of generality, that $x \| a_{n+2}$. Let $j_{0}$ be the maximal index $j$ with $x>a_{j_{0}}$, it follows that $\left\{a_{j_{0}}, \ldots, a_{n+2}\right\} \cup\left\{x, b_{j_{0}+1}, \ldots, b_{n+3}\right\} \cup\{c, d\}$ generates $\mathbf{E}_{m}^{d}$ where $m<n$. Now suppose, that $x<d$. Thus $b_{1}, b_{n+3} \in \operatorname{Inc}(x)$. Note, that if $x>a_{j}$ for some $j \neq i-1, i$, then $B(P)$ contains $\mathbf{E}_{m}^{d}$ for some $m<n$. Therefore, we may assume the existence of elements $b_{2}^{\ell}, b_{3}^{\ell}, \ldots, b_{n+2}^{\ell}$ in $P$.

Finally, from Lemma 3 we obtain elements $b_{1}^{\ell}, b_{n+3}^{\ell}, c^{\ell}, d^{\ell}$ and $b_{1}^{u}, b_{n+3}^{u}, c^{u}$. The bipartite order corresponding these elements forms a copy of $\mathcal{F}_{n}$ in $P$.

Case 12. $B(P)$ contains $\mathbf{B}$.

Suppose, that $a$ is not up-made. If there is an up-made element $a^{\prime}<a$ with $\left|\operatorname{Succ}\left(a^{\prime}\right) \cap\left\{b_{1}, b_{2}, b_{3}\right\}\right|=1$ then $B(P)$ contains $\mathbf{E}_{0}^{d}$. Otherwise, there are up-made elements $a_{1}, a_{2}, a_{3} \leq a$ with $a_{i} \| b_{i}$ and $a_{i} \| a_{j}$ for $i=1,2,3$ and $j \neq i$. However, $\left\{a_{1}, a_{2}, a_{3}, b_{1}, b_{2}, b_{3}\right\}$ generate a crown $\mathbf{A}_{3}$ in this case. We, therefore, may assume an 
element $a^{u}$.

Next, choose a low-made element $c_{1}^{\prime} \geq c_{1}$ with $b_{2}, c_{2} \in \operatorname{Inc}\left(c_{1}^{\prime}\right)$. If $c_{1}^{\prime}>c_{3}$, then $B(P)$ contains $\mathbf{C}^{d}$. So we assume $c_{1}^{\prime} \| c_{3}$. If $c_{1}^{\prime}>b_{3}$, then $B(P)$ contains $\mathbf{C X}_{1}$, so we may assume that $c_{1}^{\prime} \| b_{3}$. This gives an element $c_{1}^{\ell}$ in $P$. By symmetry, we may also assume elements $c_{2}^{\ell}$ and $c_{3}^{\ell}$.

From Lemma 3 we obtain elements $b_{1}^{\ell}, b_{2}^{\ell}, b_{3}^{\ell}$ and $b_{1}^{u}, b_{2}^{u}, b_{3}^{u}$. The bipartite order corresponding to these elements forms a copy of $\mathcal{O}_{1}^{d}$ in $P$.

Case 13. $B(P)$ contains $\mathbf{I}_{n}$.

We also assume that $B(P)$ does not contain a copy of $\mathbf{I}_{m}$ when $m<n$.

Choose an integer $i$ with $2 \leq i \leq n+2$ and a low-made element $x \geq b_{i}$ with $x \| c$. Let $j_{1}\left(j_{2}\right)$ be the minimal (maximal) integer with $x>a_{j_{1}}\left(a_{j_{2}}\right)$ and suppose that $j_{2} \neq j_{1}+1$.

Assume, that $x<d_{1}$ and $x<d_{2}$. It follows that $b_{1}, b_{n+3} \in \operatorname{Inc}(x)$ and $B(P)$ contains a copy of $\mathbf{I}_{m}$ for $m<n$.

If $x \| d_{2}$ and $j_{2}<n+2$, then $\left\{a_{j_{2}}, \ldots, a_{n+2}\right\} \cup\left\{x, b_{j_{2}+1}, \ldots, b_{n+3}\right\} \cup\left\{c, d_{2}\right\}$ is a copy of $\mathbf{E}_{m}$ for some $m<n$. We, therefore, assume that $j_{2}=n+1$. If $x<d_{1}$, then $\left\{a_{n+2}, b_{n+3}, d_{2}, c, d_{1}, x\right\}$ generate a copy of $\mathbf{D}$ in $B(P)$. Otherwise, if $x \| d_{1}$, then, by symmetry, we may also assume $x>a_{1}$. If $x \ngtr b_{1}$, then $\left\{a_{n+2}, a_{1}, b_{1}, c, d_{1}, d_{2}, x\right\}$ generate a copy of $\mathbf{F X}_{2}$ in $B(P)$. Again, by symmetry, we now assume $x>b_{1}$ and $x>b_{n+3}$, but then $\left\{b_{1}, d_{1}, c, d_{2}, b_{n+3}, x\right\}$ generates a crown $\mathbf{A}_{3}$ in $B(P)$. Hence, we may assume elements $b_{i}^{u}$ for $2 \leq i \leq n+2$ in $P$.

Now, let $i$ be an integer with $1 \leq i \leq n+2$. Choose an up-made element $a_{i}^{\prime} \leq a_{i}$ with $c \in \operatorname{Inc}\left(a_{i}^{\prime}\right)$. If $b_{1}>a_{i}^{\prime}$ and $b_{n+3}>a_{i}^{\prime}$, then $\left\{a_{i}^{\prime}, b_{n+3}, d_{2}, c, d_{1}, b_{1}\right\}$ generate a copy of $\mathbf{D}$ in $B(P)$. So we may assume, without loss of generality, that $a_{i}^{\prime} \| b_{n+3}$. It follows easily, that $B(P)$ contains $\mathbf{I}_{m}$ where $0 \leq m<n$, whenever there exists an integer $j$ with $b_{j}>a_{i}^{\prime}$ and $j \neq i, i+1$.

From Lemma 3 we obtain elements $b_{1}^{\ell}, b_{n+3}^{\ell}, c^{\ell}$ and $b_{1}^{u}, b_{n+3}^{u}, c^{u}, d_{1}^{u}, d_{2}^{u}$ in $P$. The bipartite order corresponding to these elements forms a copy of $\mathcal{I}_{n+1}$ in $P$.

Case 14. $B(P)$ contains $\mathbf{G}_{n}$.

We also assume, that $B(P)$ does not contain a copy of $\mathbf{G}_{m}, \mathbf{J}_{m}$ or $\mathbf{H}_{m}$ when $m<n$.

We first show, that we may assume an element $a_{i}^{\ell}$ when $2 \leq i \leq n+2$. Choose a low-made element $x \geq a_{i}$ with $b_{i-1}, b_{i} \in \operatorname{Inc}(x)$. Note, that from $x \| b_{i-1}$ it follows that $x \ngtr a_{j}$ and $x \ngtr b_{j}$ for all $i<j \leq n+3$.

Assume, that $x>c$ and note that this implies $x \| a_{j}$ when $i<j \leq n+3$ and $x \| b_{j}$ when $i-1 \leq j<n+3$. Let $i \neq n+2$. If $x<b_{n+3}$, then $\left\{a_{i}, a_{i+1}, \ldots, a_{n+3}\right\} \cup$ $\left\{b_{i}, b_{i+1}, \ldots, b_{n+3}\right\} \cup\{x\}$ forms a copy of $\mathbf{G}_{m}$ for some $m<n$. Otherwise, if $x \| b_{n+3}$, then the same set together with $c$ generates $\mathbf{J}_{m}$ for some $m<n$.

Now, let $i=n+2$. If $x<b_{n+3}$, then $\left\{b_{n+3}, b_{n+2}, b_{n+1}, x, a_{n+2}, a_{n+3}\right\}$ forms a copy of 
$\mathbf{D}^{d}$. Otherwise, if $x \| b_{n+3}$, then the same set together with $c$ generates $\mathbf{C X}_{3}^{d}$. Therefore, we may assume, that $x \| c$ and we have found an element $a_{i}^{\ell}$ in $P$.

We now show, that we may assume an element $b_{i}^{\ell}$ when $2 \leq i \leq n+2$. Choose a low-made element $x \geq b_{i}$ with $a_{i}, a_{i+1} \in \operatorname{Inc}(x)$.

Suppose, that $x>c$. If $i>2$, then $\left\{a_{1}, \ldots, a_{i}\right\} \cup\left\{b_{1}, \ldots, b_{i-1}, x\right\} \cup\{c\}$ forms a copy of $\mathbf{G}_{m}$ for some $m<n$. Otherwise, if $i=2$, then $\left\{a_{1}, a_{2}, a_{3}, b_{1}, x, c\right\}$ generate $\mathbf{D}$. Therefore, $x \| c$ and we have found an element $b_{i}^{\ell}$ in $P$.

We have shown, that we may assume elements $a_{i}^{\ell}$ and $b_{i}^{\ell}$ for all $2 \leq i \leq n+2$. By duality, we may as well assume elements $a_{i}^{u}$ and $b_{i}^{u}$ in $P$ for all $2 \leq i \leq n+2$. Finally, from Lemma 3 we obtain elements $a_{n+3}^{\ell}, b_{1}^{\ell}, b_{n+3}^{\ell}, c^{\ell}$ and $a_{1}^{u}, a_{n+3}^{u}, b_{1}^{u}, c^{u}$. The bipartite order corresponding to these elements forms a copy of $\mathcal{G}_{n}$ in $P$.

Case 15. $B(P)$ contains $\mathbf{J}_{n}$.

We also assume, that $B(P)$ does not contain a copy of $\mathbf{G}_{m}, \mathbf{J}_{m}$ or $\mathbf{H}_{m}$ when $m<n$.

In complete analogy with Case 14, we may assume elements $a_{i}^{\ell}$ in $P$ when $2 \leq i \leq$ $n+2$.

We now show, that we may assume an element $b_{i}^{\ell}$ when $2 \leq i \leq n+2$. Choose a low-made element $x \geq b_{i}$ with $a_{i}, a_{i+1} \in \operatorname{Inc}(x)$.

Suppose, that $x>c$. If $i>2$ and $x>d$, then $\left\{a_{1}, \ldots, a_{i}\right\} \cup\left\{b_{1}, \ldots, b_{i-1}, x\right\} \cup\{c\}$ forms a copy of $\mathbf{G}_{m}$ for some $m<n$. If $x \| d$, then the same set together with $d$ generates $\mathbf{J}_{m}$ for some $m<n$.

If $i=2$ and $x>d$, then $\left\{a_{1}, a_{2}, a_{3}, b_{1}, x, d\right\}$ generate $\mathbf{D}$. If $x \| d$, then $\left\{a_{1}, a_{2}, a_{3}, b_{1}, x, c, d\right\}$ generate $\mathbf{C X}_{3}$. The contradiction shows that $x \| c$.

We have shown, that we may assume elements $a_{i}^{\ell}$ and $b_{i}^{\ell}$ for all $2 \leq i \leq n+2$. By duality, we may as well assume elements $a_{i}^{u}$ and $b_{i}^{u}$ for all $2 \leq i \leq n+2$ in $P$. Finally, from Lemma 3 we obtain elements $a_{n+3}^{\ell}, b_{1}^{\ell}, b_{n+3}^{\ell}, c^{\ell}$ and $a_{1}^{u}, a_{n+3}^{u}, b_{1}^{u}, c^{u}$. The bipartite order corresponding to these elements forms a copy of $\mathcal{G}_{n}$ in $P$.

Case 16. $B(P)$ contains $\mathbf{H}_{n}$.

We also assume that $B(P)$ does not contain a copy of $\mathbf{H}_{m}$ when $m<n$, nor $\mathbf{G}_{m}$ or $\mathbf{J}_{m}$ when $m \leq n$.

We first show, that we may assume an element $a_{i}^{\ell}$ when $1 \leq i \leq n+2$. Choose a low-made element $x \geq a_{i}$ with $b_{i}, b_{i+1} \in \operatorname{Inc}(x)$.

Assume, that $x>c$. If $i \geq 3$, then $\left\{a_{1}, \ldots a_{i-1}, x\right\} \cup\left\{b_{1}, \ldots, b_{i}\right\} \cup\{c\}$ forms a copy of $\mathbf{G}_{m}$ for some $m<n$. Otherwise, if $i=1,2$, then $\left\{b_{1}, b_{2}, b_{3}, a_{1}, x, c\right\}$ forms a copy of D.

Assume, that $x>d$. Let $i \neq n$. If $x<b_{n+3}$, then $\left\{a_{i}, \ldots, a_{n+2}\right\} \cup\left\{b_{i+1}, \ldots, b_{n+3}\right\} \cup$ $\{x\}$ forms a copy of $\mathbf{G}_{m}$ for some $m<n$. Otherwise, if $x \| b_{n+3}$, then the same set together with $c$ generates $\mathbf{J}_{m}$ for some $m<n$.

If $i=n+1$ and $x<b_{n+3}$, then $\left\{b_{n+3}, b_{n+2}, b_{n+1}, x, a_{n+1}, a_{n+2}\right\}$ forms a copy of $\mathbf{D}^{d}$. Otherwise, if $x \| b_{n+3}$, then the same set together with $d$ generates $\mathbf{C X}_{3}^{d}$. 
If $i=n+2$, then $\left\{b_{n+1}, b_{n+2}, b_{n+3}, d, x, a_{n+2}\right\}$ forms a copy of $\mathbf{D}$.

Therefore, we may assume $c, d \in \operatorname{Inc}(x)$ and have thus found an element $a_{i}^{\ell}$.

We now show, that we may assume an element $b_{i}^{\ell}$ when $2 \leq i \leq n+2$. Choose a low-made element $x \geq b_{i}$ with $a_{i-1}, a_{i} \in \operatorname{Inc}(x)$.

Suppose, that $x>c$ and $x>d$, then $\left\{b_{1}, a^{\prime}, b_{n+3}, d, x, c\right\}$ forms a copy of $\mathbf{D}$ where $a^{\prime}$ is one of $a_{i-1}$ or $a_{i}$.

Suppose $x>c$ and $x \| d$, it follows, that $x \| a_{j}$ and $x \| b_{j}$ when $j>i$. If $i<n+2$ then $\left\{a_{i}, \ldots, a_{n+2}\right\} \cup\left\{b_{i} \ldots b_{n+3}\right\} \cup\{x, d\}$ generates $\mathbf{H}_{m}$ for some $m<n$. If $i=n+2$, then $\left\{a_{n+1}, a_{n+2}, b_{n+1}, b_{n+2}, b_{n+3}, d, x\right\}$ generates $\mathbf{C X}_{3}^{d}$.

Finally, let $x>d$ and $x \| c$. If $i>2$, then $\left\{a_{1}, \ldots, a_{i-1}\right\} \cup\left\{b_{1} \ldots b_{i-1}, x\right\} \cup\{c, d\}$ generates $\mathbf{H}_{m}$ for some $m<n$. If $i=2$, then depending on $x<b_{n+3}$ or $x \| b_{n+3}$, a copy of either $\mathbf{C X}_{1}$ or $\mathbf{C X}_{2}$ is formed by $\left\{a_{1}, a_{n+2}, b_{1}, b_{n+3}, c, d, x\right\}$.

We, therefore, may assume $c, d \in \operatorname{Inc}(x)$ and have found elements $b_{i}^{\ell}$ for $2 \leq i \leq n+2$.

If $b_{n+3}$ is not low-made, then choose a low-made element $b_{n+3}^{\prime}>b_{n+3}$ with $a_{n+2} \| b_{n+3}^{\prime}$. If $b_{n+3}^{\prime}>c$, then $\left\{a_{1}, a_{n+2}, b_{1}, b_{n+3}, c, d, x\right\}$ generate a copy of $\mathbf{F X}_{1}$. Therefore, we may assume an element $b_{n+3}^{\ell}$ in $P$.

We have shown, that we may assume elements $a_{i}^{\ell}$ for $1 \leq i \leq n+2$ and $b_{i}^{\ell}$ for $2 \leq i \leq n+3$. Dually, we may assume elements $a_{i}^{u}$ for $1 \leq i \leq n+2$ and $b_{i}^{u}$ in $P$ for $1 \leq i \leq n+2$. Finally, from Lemma 3 we obtain elements $c^{\ell}, d^{\ell}$ and $c^{u}, d^{u}$. The bipartite order corresponding to these elements forms a copy of $\mathcal{H}_{n}$ in $P$.

2.2. 3-interval irreducible partial stacks. We begin this part with an analysis of the relation between the interval dimension of a bipartite order $P$ and the interval dimension of partial stacks of $P$.

Lemma 5. Let $P=(X,<)$ be a bipartite poset and $P^{\prime}=\left(X,<^{\prime}\right)$ be a partial stack of $P$, then $\operatorname{Idim} P^{\prime} \geq \operatorname{Idim} P$.

Proof. Let $I_{1}^{\prime}, \ldots, I_{k}^{\prime}$ be a interval-realizer of $P^{\prime}$. Now transform each of the interval orders $I_{i}^{\prime}$. Extend the intervals of elements in $\operatorname{Min}(P)$ to the left to the leftmost endpoint of an interval in $I_{i}^{\prime}$, symmetrically, extend the intervals of elements in $\operatorname{Max}(P)$ to the right to the rightmost endpoint of an interval in $I_{i}^{\prime}$. From this transformation we obtain a new family $I_{1}, \ldots, I_{k}$ of interval orders. This family is an interval-realizer of $P$. Hence, $\operatorname{Idim} P^{\prime} \geq \operatorname{Idim} P$ as claimed.

As a consequence we can now sharpen the result of Theorem 4.

Theorem 7. If $P=\left(X,<_{P}\right)$ is a 3-interval irreducible poset then $P$ is a reduced partial stack of some bipartite 3-interval irreducible poset.

Proof. From Theorem 4 we know that $P$ contains a reduced partial stack of some bipartite 3-interval irreducible poset $R=\left(Y,<_{R}\right)$. Therefore, there are sets $X_{1}, X_{2} \subseteq$ 
$X$, such that $\left(X_{1} \times X_{2}\right) \cap<_{P} \cong<_{R}$. Let $R^{*}$ be the poset induced by $P$ on $X_{1} \cup X_{2}$. This poset is a reduced partial stack of $R$.

From Lemma 5 we obtain $\operatorname{Idim} R^{*} \geq \operatorname{Idim} R=3$. On the other hand, $R^{*}$ is a suborder of $P$, hence, $3=\operatorname{Idim} P \geq \operatorname{Idim} R^{*}$. The irreducibility of $P$ implies that $X=X_{1} \cup X_{2}$ and, therefore, $P=R^{*}$.

Lemma 6. Let $P=(X,<)$ be a bipartite order, then $\operatorname{Idim} P=\operatorname{Idim} \operatorname{Stack}(P)$.

Proof. From Lemma 5 we obtain $\operatorname{Idim} \operatorname{Stack}(P) \geq \operatorname{Idim} P$. As noted in the Introduction and proved in Theorems 1 and $2 \operatorname{Idim} P=\operatorname{dim} \operatorname{Stack}(P)$. Together this gives $\operatorname{Idim} \operatorname{Stack}(P) \geq \operatorname{dim} \operatorname{Stack}(P)$, the converse of this inequality is trivially valid for every order.

Theorem 7 shows, that a classification of 3 -interval irreducible posets amounts in work with partial stacks of bipartite orders. We, therefore, require a suitable notation for these objects.

Remark. An intuitive approach to partial stacks of a (connected) bipartite poset $P=\left(X,<_{P}\right)$ would be the following: Let $A=\operatorname{Min}(P)$ and $B=\operatorname{Max}(P)$. Consider the interval representation of $P$ on $B(P)=\left(Y,<_{Q}\right)$. The intervals of elements $a \in A$ have $L(a)=0$ and for $b \in B$ have $U(b)=1$. These are called free-ends. This name is motivated by the observation: Suppose two functions $L^{\prime}, U^{\prime}: X \rightarrow Y$ are given, such that, $0 \leq L^{\prime}(a)<U(a)$ and $U^{\prime}(a)=U(a)$ for all $a \in A$ and $L^{\prime}(b)=L(b)$ and $1 \geq U^{\prime}(b)>L(b)$ for all $b \in B$. The intervals $\left(L^{\prime}(x), U^{\prime}(x)\right)$ then define an ordering $P^{\prime}=\left(X,<_{P}^{\prime}\right)$, such that, $(A \times B) \cap<_{P}=(A \times B) \cap<_{P}^{\prime}$, i.e., a partial stack of $P$. For an example see Figure 3.

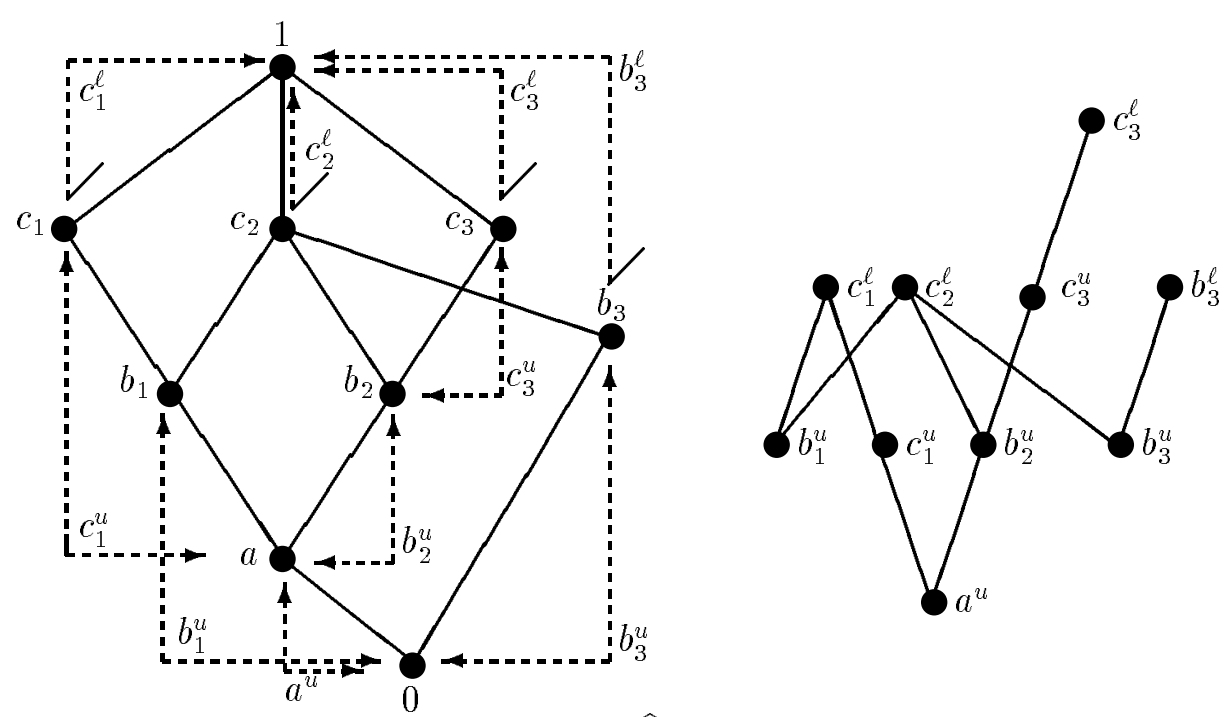

Figure 3. A placement of the free-ends in $\widehat{\mathbf{C}}$ and the corresponding partial stack of $\mathrm{O}_{2}$. 
Unfortunately the above construction will not lead to all partial stacks. As an example note that there are partial stacks of $\mathcal{O}_{2}$ with $c_{3}^{u}>b_{2}^{u}$ but $c_{3}^{u}|| a^{u}$. These partial stacks are not representable on $B\left(\mathcal{O}_{2}\right)$. Therefore we require a more formal description of partial stacks.

Let $\operatorname{Succ}_{S P}(x)\left(\operatorname{Pred}_{S P}(x)\right)$ denote the successor sets (predecessor sets) of $x$ in $\operatorname{Stack}(P)$. To describe a partial stack $Q=\left(X,<_{Q}\right)$ of a bipartite poset $P=\left(X,<_{P}\right)$ we will, henceforth, use sets

$\operatorname{Succ}(x)$ with $\operatorname{Succ}(x) \subseteq \operatorname{Succ}_{S P}(x)$ for each $x \in \operatorname{Max}(P)$,

$\operatorname{Pred}(x)$ with $\operatorname{Pred}(x) \subseteq \operatorname{Pred}_{S P}(x)$ for each $x \in \operatorname{Min}(P)$.

If such a family of sets is given, then the partial stack $Q$ of $P$ denoted by the family is the transitive closure of the union of all relations occurring in these sets together with the relations of $P$.

Lemma 5 and Lemma 6 might suggest that $\operatorname{Idim} P=\operatorname{Idim} Q$ for every partial stack $Q$ of $P$. This, however, is far from truth as the next lemma shows.

Lemma 7. For every integer $n$ there is a bipartite interval order $I$, such that, every bipartite poset $R$ on $n$ elements is a suborder of some partial stack $I_{R}$ of $I$.

Proof. As $I$ we take the interval order with $2 n$ minimal elements $\left\{a_{1}, \ldots, a_{2 n}\right\}$ and with $2 n$ maximal elements $\left\{b_{1}, \ldots, b_{2 n}\right\}$. The relations of $I$ are: $a_{i}<b_{j}$ exactly if $i \leq j$. Now fix a bipartite $n$-element poset $R$ with minimal elements $\left\{x_{1}, \ldots, x_{k}\right\}$ and maximal elements $\left\{y_{1}, \ldots, y_{n-k}\right\}$. A partial stack $I_{R}$ containing $R$ is given by:

$$
\begin{aligned}
& \operatorname{Pred}\left(a_{i}\right)=\emptyset \text { for } 1 \leq i \leq k \\
& \operatorname{Pred}\left(a_{i}\right)=\left\{a_{1}, \ldots, a_{k}\right\} \cup\left\{b_{j}: x_{j} \leq_{R} y_{i}\right\} \quad \text { for } k<i \leq 2 n \\
& \operatorname{Succ}\left(b_{i}\right)=\left\{b_{k+1}, \ldots, b_{2 n}\right\} \cup\left\{a_{k+j}: x_{i} \leq_{R} y_{j}\right\} \quad \text { for } 1 \leq i \leq k \\
& \operatorname{Succ}\left(b_{i}\right)=\emptyset \text { for } k<i \leq 2 n
\end{aligned}
$$

Note, that $B(I)$ is a chain of length $2 n+2$, while $B\left(I_{R}\right)$ consists of a chain of length $2 n+3$, where the element $k+1$ has been substituted by a copy of $B(R)$ without 0 and 1.

As a consequence we obtain, that the gap between the interval dimension of a bipartite poset $P$ and and the interval dimension of partial stacks of $P$ can be arbitrarily high.

We now turn to the partial stacks of $\mathcal{G}_{m}$ and $\mathcal{H}_{m}$. There will be some indications, that the complete classification of 3-interval irreducible posets among the reduced partial stacks of these two orders might be intractable.

Theorem 8. Every bipartite poset $R$ is a suborder of a partial stack $Q_{R}$ of $\mathcal{G}_{m}\left(\mathcal{H}_{m}\right)$. Moreover, every bipartite 2-dimensional poset $R$ is a suborder of an 3-interval irreducible partial stack of $\mathcal{G}_{m}\left(\mathcal{H}_{m}\right)$. 
Proof. We prove the theorem for partial stacks of $\mathcal{H}_{m}$. The idea behind the proof is the same as in the proof of Lemma 7 .

Let $R$ be a bipartite $n$-element poset with minimal elements $\left\{x_{1}, \ldots, x_{k}\right\}$ and maximal elements $\left\{y_{1}, \ldots, y_{n-k}\right\}$. Also, let a copy of $\mathcal{H}_{n-2}$ be given on the set $\left\{a_{1}^{\ell}, \ldots, a_{n}^{\ell}\right\} \cup\left\{b_{2}^{\ell}, \ldots, b_{n+1}^{\ell}\right\} \cup\left\{c^{\ell}, d^{\ell}\right\} \cup\left\{a_{1}^{u}, \ldots, a_{n}^{u}\right\} \cup\left\{b_{1}^{u}, \ldots, b_{n}^{u}\right\} \cup\left\{c^{u}, d^{u}\right\}$. The relations of $\mathcal{H}_{n-2}$ are: $x^{\ell}>y^{u}$ exactly if $x \geq y$ in $\mathbf{H}_{n-2}$. A partial stack $Q_{R}$ of $\mathcal{H}_{n-2}$ containing $R$ is given by:

$$
\begin{aligned}
\operatorname{Pred}\left(a_{i}^{u}\right)= & \emptyset \text { for } 1 \leq i \leq k \\
\operatorname{Pred}\left(a_{i}^{u}\right)= & \left\{a_{j}^{u}: 1 \leq j \leq k\right\} \cup\left\{b_{j}^{u}: 1 \leq j \leq k\right\} \cup\left\{a_{j}^{\ell}: x_{j} \leq_{R} y_{i}\right\} \\
& \text { for } k<i \leq n \\
\operatorname{Succ}\left(a_{i}^{\ell}\right)= & \left\{a_{j}^{\ell}: k<j \leq n\right\} \cup\left\{b_{j}^{\ell}: k<j \leq n+1\right\} \cup\left\{a_{j}^{u}: x_{i} \leq_{R} y_{j}\right\} \\
& \text { for } 1<i \leq k \\
\operatorname{Succ}\left(a_{i}^{\ell}\right)= & \emptyset \text { for } k<i \leq n
\end{aligned}
$$

All other elements $z^{\ell}$ have $\operatorname{Succ}\left(z^{\ell}\right)=\emptyset$ and all other elements $z^{u}$ have $\operatorname{Pred}\left(z^{u}\right)=\emptyset$. This gives a partial stack $Q_{R}$ of $\mathcal{H}_{m}$ and the poset generated by the elements $\left\{\boldsymbol{a}_{i}^{\ell}: 1<\right.$ $i \leq k\} \cup\left\{a_{i}^{u}: k<i \leq n\right\}$ is isomorphic to $R$.

Let $\mathbf{H}_{n-2}^{+}$be isomorphic to $\mathbf{H}_{n-2}$ together with a new element $\boldsymbol{\epsilon}$ such that Pred $(\epsilon)=$ $\left\{a_{1}, \ldots, a_{k}, b_{1}, \ldots, b_{k}\right\}$ and $\operatorname{Succ}(e)=\left\{a_{k+1}, \ldots, a_{n}, b_{k+2}, \ldots, b_{n+1}\right\}$. The order $B\left(Q_{R}\right)$ is obtained by substituting $e$ in $\mathbf{H}_{n-2}^{+}$by a copy of $B(R)$ without 0 and 1 .

If $R$ is 2-dimensional, then we claim that $Q_{R}$ is 3 -interval irreducible. To verify this it suffices to consider the effect of point deletion in $Q_{R}$ only on the essentially 3-dimensional part of $B\left(Q_{R}\right)$, i.e., on $\mathbf{H}_{n-2}^{+}$

Remark. Let $\mathbf{H}_{m}=(X,<)$ and let the completion by cuts of $\mathbf{H}_{m}$ be denoted by $\mathbf{L}\left(\mathbf{H}_{m}\right)=(X \cup Y,<)$. A large class of 3-interval irreducible reduced partial stacks of $\mathcal{H}_{m}$ can be characterized by the following two properties:

(1) $B(Q)$ is an order isomorphic to $\mathbf{L}\left(\mathbf{H}_{m}\right)$ with elements of $Y$ substituted by (possibly empty) 2-dimensional posets.

(2) If $x$ is $\ell$-labeled ( $u$-labeled) in $\mathbf{H}_{m}$, then there is a unique element $y \in Q$ with $L(y)=x(U(y)=x)$.

Similar properties describe a large class of 3-interval irreducible reduced partial stacks of $\mathcal{G}_{m}$.

However, there are more complex examples among the 3 -irreducible partial stacks of $\mathcal{G}_{m}\left(\mathcal{H}_{m}\right)$. Here is an example.

\section{Example.}

Let $\mathcal{G}_{0}$ consist of the elements $\left\{a_{2}^{\ell}, a_{3}^{\ell}, b_{1}^{\ell}, b_{2}^{\ell}, b_{3}^{\ell}, c^{\ell}\right\} \cup\left\{a_{1}^{u}, a_{2}^{u}, a_{3}^{u}, b_{1}^{u}, b_{2}^{u}, c^{u}\right\}$ and relations 
$x^{\ell}>y^{u}$ exactly if $x \geq y$ in $\mathbf{G}_{0}$. Let $Q$ be the partial stack of $\mathcal{G}_{0}$ defined by:

$$
\begin{aligned}
\operatorname{Succ}\left(a_{2}^{\ell}\right) & =\left\{a_{3}^{u}, a_{3}^{\ell}, b_{3}^{\ell}\right\} \\
\operatorname{Succ}\left(b_{1}^{\ell}\right) & =\left\{a_{3}^{\ell}, b_{2}^{u}, b_{2}^{\ell}, b_{3}^{\ell}\right\} \\
\operatorname{Pred}\left(a_{3}^{u}\right) & =\left\{a_{2}^{\ell}, a_{2}^{u}, a_{1}^{u}, b_{1}^{u}\right\} \\
\operatorname{Pred}\left(b_{2}^{u}\right) & =\left\{a_{1}^{u}, b_{1}^{u}, b_{1}^{\ell}\right\}
\end{aligned}
$$

All other elements $x^{\ell}$ have $\operatorname{Succ}\left(x^{\ell}\right)=\emptyset$ and all other elements $x^{u}$ have $\operatorname{Pred}\left(x^{u}\right)=\emptyset$. This gives a partial stack $Q$ of $\mathcal{G}_{0}$ which does not fall into the class of the previous remark.

With these partial results we leave the classification of 3-interval irreducible reduced partial stacks of $\mathcal{G}_{m}$ and $\mathcal{H}_{m}$ as an open problem.

Problem 1. Characterize the 3-interval irreducible reduced partial stacks of $\mathcal{G}_{m}$ and $\mathcal{H}_{m}$.

We now turn to the partial stacks of the other bipartite 3-interval irreducible posets. With the next theorem we classify the 3 -interval irreducible orders among them.

Theorem 9. Every partial stack of $\mathcal{A}_{n}, \mathcal{O}_{1}, \mathcal{O}_{2}, \mathcal{O}_{3}, \mathcal{E}_{n}, \mathcal{I}_{n}, \mathcal{F}_{0}$ for every integer $n \geq 0$ and every partial stack of $\mathcal{F}_{n}(n>0)$ which has neither $\left(a_{n+2}^{\ell}<b_{n+2}^{\ell}\right.$ and $\left.a_{n+2}^{\ell} \| \epsilon^{\ell}\right)$ nor $\left(b_{1}^{u}>a_{1}^{u}\right.$ and $\left.b_{1}^{u} \| c^{u}\right)$ is a 3 -interval irreducible poset.

Proof. The argument is again divided into a series of cases. As criterion for the irreducibility of a candidate partial stack $Q$ we use a technical lemma.

Lemma 8. Let $P=\left(X,<_{P}\right)$ be a poset of interval dimension 3. Let $Q=\left(Y,<_{Q}\right)$ be 3-irreducible and let $P$ have an interval representation on $\hat{Q} . P$ is 3 -interval irreducible if the following three conditions are satisfied:

(1) For every element $y$ of $Q$ one of three cases applies

(a) y has two essential labels.

(b) $y$ has an essential label $\ell$ and a unique element $y^{\prime}$ in $\hat{Q}$ is covered by $y$.

(c) y has an essential label $u$ and a unique element $y^{\prime \prime}$ in $\hat{Q}$ is covering $y$.

(2) Every element of $Q$ has an essential label and every essential label of $Q$ is satisfied by exactly one element of $P$.

(3) Every element of $P$ satisfies at least one essential label of $Q$.

Proof. We have to show that for every $x \in X$ the interval dimension of $P_{x}$ is at most 2 . To do this we exhibit a poset $Q(x)$ of dimension 2 admitting an interval representation of $P_{x}$.

Let $x \in X$ and let $y_{x}$ be an element of $Q$ such that $x$ satisfies an essential label at $y_{x}$. By duality we may suppose, that $x$ satisfies the label $\ell$ at $y_{x}$. We distinguish two cases. 
Suppose, that $y_{x}$ has two essential labels. Let $R=\left(Z,<_{R}\right)$ be the bipartite 3 interval irreducible poset corresponding to the essential labels of $Q$. By definition, $R_{y_{x}^{\ell}}$ has interval dimension 2 and, hence, $\operatorname{dim} B\left(R_{y_{x}^{\ell}}\right)=2$. We claim that $P_{x}$ has an interval representation on $B\left(R_{y_{x}^{e}}\right)$. Let $x^{\prime}$ be any element of $P_{x}$, and denote the two endpoints of the interval of $x^{\prime}$ on $\widehat{Q}$ by $L\left(x^{\prime}\right)=y_{1}$ and $U\left(x^{\prime}\right)=y_{2}$. If $y_{1}=0$ or $y_{2}=1$, then the mappings $L^{*}, U^{*}: P_{x} \rightarrow B\left(R_{y_{x}^{\ell}}\right)$, also map the corresponding interval end to 0 or 1. Let the essential labels at $y_{1}, y_{2}$ be $\rho_{1}, \rho_{2}$. In $R$ we find elements $y_{1}^{\rho_{1}}$ and $y_{2}^{\rho_{2}}$ with $y_{1}^{\rho_{1}} \neq y_{x}^{\ell}$ and $y_{2}^{\rho_{2}} \neq y_{x}^{\ell}$. Now, let $L^{*}\left(x^{\prime}\right)=L\left(y_{1}^{\rho_{1}}\right)$ if $\rho_{1}=\ell$ and $L^{*}\left(x^{\prime}\right)=U\left(y_{1}^{\rho_{1}}\right)$ if $\rho_{1}=u$. Similarly, $U^{*}\left(x^{\prime}\right)=L\left(y_{1}^{\rho_{1}}\right)$ if $\rho_{1}=\ell$ and $U^{*}\left(x^{\prime}\right)=U\left(y_{1}^{\rho_{1}}\right)$ if $\rho_{1}=u$. It is easy to verify that the mappings $L^{*}, U^{*}: P_{x} \rightarrow B\left(R_{y_{x}^{e}}\right)$ indeed define an interval representation of $P_{x}$.

Next, suppose that there is no essential label at $y_{x}$. Our assumptions imply, that there is a unique element $y_{x}^{\prime}$ covered by $y_{x}$. We claim that $P_{x}$ has an interval representation on $\widehat{Q_{y_{x}}}$. Since $Q$ is 3 -irreducible we conclude that $\operatorname{dim} \widehat{Q_{y_{x}}}=2$ and, hence, that the interval dimension of $P_{x}$ is 2 . As mappings $L^{*}, U^{*}: P_{x} \rightarrow \widehat{Q_{y_{x}}}$ choose the mappings induced by $L, U: P \rightarrow \widehat{Q}$, except for elements $x^{\prime}$ with $U\left(x^{\prime}\right)=y_{x}$. For such an element let $U^{*}\left(x^{\prime}\right)=y_{x}^{\prime}$. Again, it is easy to verify that the mappings $L^{*}, U^{*}: P_{x} \rightarrow \widehat{Q_{y_{x}}}$ define an interval representation of $P_{x}$.

Case 1. $P$ is a partial stack of $\mathcal{A}_{n}$.

Since $\operatorname{Stack}\left(\mathcal{A}_{n}\right)=\mathcal{A}_{n}$ we obtain $Q=\mathcal{A}_{n}$ and $P$ is 3 -interval irreducible.

Case 2. $P$ is a partial stack of $\mathcal{O}_{1}$.

The partial stacks of $\mathcal{O}_{1}$ differ only in $\operatorname{Succ}\left(b_{i}^{\ell}\right)$ for $i=1,2,3$ which is either empty or $\left\{c_{i}^{\ell}\right\}$. An interval representation on $\widehat{\mathbf{B}}$ is obtained if we let $U\left(b_{i}^{\ell}\right)=c_{i}$ when $\operatorname{Succ}\left(b_{i}^{\ell}\right)=$ $\left\{c_{i}^{\ell}\right\}$ and else $U\left(b_{i}^{\ell}\right)=1$. These representations satisfy the conditions of Lemma 8 and, hence, $P$ is 3 -interval irreducible.

Case 3. $P$ is a partial stack of $\mathcal{O}_{2}$.

If $\operatorname{Pred}\left(c_{1}^{u}\right)$ is one of $\left\{a^{u}, b_{1}^{u}\right\}$ or $\left\{a^{u}\right\}$ or $\emptyset$ and $\operatorname{Pred}\left(c_{3}^{u}\right)$ is one of $\left\{a^{u}, b_{2}^{u}\right\}$ or $\left\{a^{u}\right\}$ or $\emptyset$, then $B(P)=\widehat{\mathbf{C}}$. Moreover, the pair $(P, \mathbf{C})$ satisfies the conditions of Lemma 8 and, therefore, $P$ is 3 -interval irreducible.

If $\operatorname{Pred}\left(c_{1}^{u}\right)$ is one of $\left\{a^{u}, b_{1}^{u}\right\}$ or $\left\{a^{u}\right\}$ or $\emptyset$ but $\operatorname{Pred}\left(c_{3}^{u}\right)=\left\{b_{2}^{u}\right\}$, then $B(P)=\widehat{\mathrm{CX}}_{1}{ }^{d}$. Again, Lemma 8 yields the 3 -interval irreducibility of $P$.

By symmetry, we may now assume that $\operatorname{Pred}\left(c_{1}^{u}\right)=\left\{b_{1}^{u}\right\}$ and $\left.\operatorname{Pred}\left(c_{3}^{u}\right)\right)=\left\{b_{2}^{u}\right\}$. Here, $B(P)=\widehat{\mathrm{CX}}_{2}^{d}$ and again, $P$ is 3-interval irreducible by Lemma 8 .

Case 4. $P$ is a partial stack of $\mathcal{O}_{3}$.

All partial stacks of $\mathcal{O}_{3}$, together with interval representations on $\widehat{\mathbf{E X}}_{2}$ can be obtained by all possible choices of $L\left(b_{1}^{u}\right) \in\left\{a_{1}, 0\right\}, L\left(b_{3}^{u}\right) \in\left\{a_{2}, 0\right\}, U\left(b_{1}^{\ell}\right) \in\{c, 1\}$ and $U\left(a_{3}^{\ell}\right) \in$ $\left\{b_{2}, 1\right\}$. With Lemma 8 we then obtain the 3 -interval irreducibility of $P$. 
Case 5. $P$ is a partial stack of $\mathcal{E}_{n}$.

All partial stacks of $\mathcal{E}_{n}$, together with interval representations on $\widehat{\mathbf{E}_{n}}$ can be obtained by all possible choices of $L\left(b_{1}^{u}\right) \in\left\{a_{1}, 0\right\}, L\left(b_{n+3}^{u}\right) \in\left\{a_{n+2}, 0\right\}$ and for $x \in\left\{b_{2}^{\ell}, \ldots, b_{n+2}^{\ell}, c^{\ell}\right\}$ either $U(x)=d$ or $U(x)=1$. These representations satisfy the conditions of Lemma 8 and, hence, $P$ is 3 -interval irreducible.

Case 6. $P$ is a partial stack of $\mathcal{I}_{n}$.

First, suppose that $n=0$ and let $\mathbf{D}^{+}$be obtained from $\mathbf{D}$ by adding a new element $d$ with $d>b_{2}$ and $d<c_{1}, c_{2}$. We claim that every partial stack of $\mathcal{I}_{0}$ has an interval representation on $\widehat{\mathrm{D}^{+}}$. It should be clear that for $i=1,3$ we find an appropriate point for $U\left(b_{i}^{\ell}\right)$ and $L\left(b_{i}^{u}\right)$ in $\widehat{\mathbf{D}^{+}}$. It remains to consider $U\left(b_{2}^{\ell}\right)$. In $\operatorname{Stack}\left(\mathcal{I}_{0}\right)$ the successor set of $b_{2}^{\ell}$ is $\left\{c_{1}^{\ell}, c_{2}^{\ell}\right\}$ and, hence, $\operatorname{Succ}_{P}\left(b_{2}^{\ell}\right) \subseteq\left\{c_{1}^{\ell}, c_{2}^{\ell}\right\}$. If $\operatorname{Succ}_{P}\left(b_{2}^{\ell}\right)=\left\{c_{1}^{\ell}, c_{2}^{\ell}\right\}$, then let $U\left(b_{2}^{\ell}\right)=d$. If $\operatorname{Succ}_{P}\left(b_{2}^{\ell}\right)=\left\{c_{i}^{\ell}\right\}$ with $i=1$ or $i=2$, then let $U\left(b_{2}^{\ell}\right)=c_{i}$. Finally, if $\operatorname{Succ}_{P}\left(b_{2}^{\ell}\right)=\emptyset$, then let $U\left(b_{2}^{\ell}\right)=1$. With a slight generalization of Lemma 8 we obtain the 3-interval irreducibility of $P$.

Now, let $n>0$ and let $\mathbf{I}_{n}^{+}$be obtained from $\mathbf{I}_{n}$ by adding a new element $e$ with $e<d_{1}, e\left\langle d_{2}, e\right\rangle c$ and $e>b_{i}$ for $i=2, \ldots, n+2$. An analysis as in the previous case shows that every partial stack of $\mathcal{I}_{n}$ has an interval representation on $\widehat{\mathbf{I}_{n}^{+}}$. (Note that $\widehat{\mathbf{I}_{n}^{+}}=\mathbf{L}\left(\mathbf{I}_{n}\right)$ ). Again, the 3-interval irreducibility of $P$ follows from the generalization of Lemma 8.

Case 7. $P$ is a partial stack of $\mathcal{F}_{0}$.

If $\operatorname{Pred}\left(b_{1}^{u}\right)$ is one of $\left\{a_{1}^{u}, c^{u}\right\}$ or $\left\{c^{u}\right\}$ or $\emptyset$ and $\operatorname{Succ}\left(a_{2}^{\ell}\right)$ is one of $\left\{b_{2}^{\ell}, e^{\ell}\right\}$ or $\left\{e^{\ell}\right\}$ or $\emptyset$, then $B(P)=\widehat{\mathbf{F}_{0}}$. Moreover, the pair $\left(P, \mathbf{F}_{0}\right)$ satisfies the conditions of Lemma 8 and, therefore, $P$ is 3 -interval irreducible.

If $\operatorname{Pred}\left(b_{1}^{u}\right)$ is one of $\left\{\boldsymbol{a}_{1}^{u}, c^{u}\right\}$ or $\left\{c^{u}\right\}$ or $\emptyset$ but $\operatorname{Succ}\left(a_{2}^{\ell}\right)=\left\{b_{2}^{\ell}\right\}$, then $B(P)=\widehat{\mathbf{F X}}_{1}{ }^{d}$. Again, Lemma 8 yields the 3 -interval irreducibility of $P$.

By symmetry we may now assume that $\operatorname{Pred}\left(b_{1}^{u}\right)=\left\{a_{1}^{u}\right\}$ and $\operatorname{Succ}\left(a_{2}^{\ell}\right)=\left\{b_{2}^{\ell}\right\}$. Here, $B(P)=\widehat{\mathbf{F X}}_{2}{ }^{d}$ and $P$ is 3 -interval irreducible by Lemma 8 .

Case 8. $\quad P$ is a partial stack of $\mathcal{F}_{n}$ with $n>0$.

Suppose that $\operatorname{Succ}_{P}\left(a_{n+2}^{\ell}\right)=\left\{b_{n+2}^{\ell}\right\}$. The bipartite subposet of $P$ with minimal elements $\left\{a_{1}^{u}, \ldots, a_{n+2}^{u}, b_{1}^{u}, d^{u}\right\}$ and $\left\{b_{1}^{\ell}, \ldots, b_{n+2}^{\ell}, d^{\ell}, e^{\ell}\right\}$ as maximal elements forms a copy of $\mathcal{E}_{n-1}$. If we remove $c^{u}$ and $a_{n+2}^{u}$ from $P$ to obtain $P^{-}$, then $P^{-}$is a partial stack of $\mathcal{E}_{n-1}$. Therefore, $P$ is not irreducible.

We may now assume that $\operatorname{Succ}_{P}\left(a_{n+2}^{\ell}\right)$ is either $\left\{b_{n+2}^{\ell}, e^{\ell}\right\}$ or $\left\{e^{\ell}\right\}$ or $\emptyset$. Dually, we may assume that $\operatorname{Pred}_{P}\left(b_{1}^{u}\right)$ is $\left\{a_{1}^{u}, c^{u}\right\}$ or $\left\{c^{u}\right\}$ or $\emptyset$. It follows that $B(P)=\widehat{\mathbf{F}_{n}}$ and with Lemma 8 that $P$ is 3 -interval irreducible. 


\section{REFERENCES}

[DuMi41] B. Dushnik and E. Miller, Partially Ordered Sets, Amer. J. Math. 63 (1941), 600-610.

[FHM91] S. FELsner, M. HaBiB AND R.H. MöHRing, On the Interplay of Interval Dimension and Dimension, Preprint 285, TU-Berlin 1991.

[Fi85] P.C. Fishiurn, Interval Orders and Interval Graphs, Wiley, New York, 1985.

[Ke77] D. Kelly, The 3-irreducible partially ordered sets, Can. J. of Math. 29 (1977) 367-383.

[KeRi75] D. Kelly and I. Rival, Planar Lattices, Can. J. of Math. 27 (1975) 636-665.

[KeTr82] D. Kelly and W.T. Trotter, Dimension Theory for Ordered Sets, in 'Ordered Sets', I. Rival ed., 171-212, D. Reidel Publishing Comp., 1982.

[Mi92] J. Mitas, Interval representations on arbitrary ordered sets, Preprint, Darmstadt 1992.

[TrMo76] W.T. Trotter and J.I. Moore, Characterization Problems for Graphs, Partially Ordered Sets, Lattices and Families of Sets, Discrete Math. 16 (1976) 361-381.

[Tr81] W.T. Trotter, Stacks and Splits of Partially Ordered Sets, Discrete Math. 35 (1981) 229-256.

[Tr91] W.T. Trotter, Combinatorics and Partially Ordered Sets: Dimension Theory, Johns Hopkins Press, 1991.

Freie Universität Berlin, Fachbereich Mathematik, Institut für Informatik, Takustr. 9, 14195 Berlin, Germany

E-mail address: felsner@inf.fu-berlin.de 